\title{
RACSAM
}

Rev. R. Acad. Cien. Serie A. Mat.

VOL. 103 (1), 2009, pp. 177-200

Matemática Aplicada / Applied Mathematics

\section{On Nonlinear Parametric Problems for $p$-Laplacian-Like Operators}

\author{
N. S. Papageorgiou and E. M. Rocha
}

\begin{abstract}
We study a nonlinear parametric problem driven by a $p$-Laplacian-like operator (which need not be homogeneous) and with a $(p-1)$-superlinear nonlinearity which satisfy weaker conditions than the Ambrosetti-Rabinowitz condition. Using critical point theory, we show that for every $\lambda>0$, the nonlinear parametric problem has a nontrivial solution. Then, by strengthening the conditions on the operator and the nonlinearity, and using variational methods together with suitable truncation techniques and tools from Morse theory, we show that, for every $\lambda>0$, the nonlinear parametric problem has three nontrivial smooth solutions.
\end{abstract}

\section{Sobre los problemas paramétricos no lineales con operadores de tipo $p$-Laplaciano}

Resumen. En este artículo estudiamos un problema paramétrico no lineal que involucra al operador de tipo $p$-Laplaciano (que en general no és homogéneo) y donde la derivada del potencial es una función $(p-1)$-superlinear que verifica una condición más débil que la conocida condición de AmbrosettiRabinowitz. Utilizando métodos variacionales, mostramos que, para todo $\lambda>0$, el problema paramétrico no lineal tiene una solución no trivial. Entonces, fortaleciendo las condiciones y usando herramientas de la teoría de Morse junto con adecuadas técnicas de truncación, mostramos que, para cada $\lambda>0$, el problema tiene tres soluciones suaves.

\section{Introduction}

Let $Z \subseteq \mathbb{R}^{N}$ be a bounded domain with a $C^{2}$-boundary $\partial Z$. We study the following nonlinear elliptic parametric problem driven by a $p$-Laplacian-like operator,

$$
\left\{\begin{array}{l}
-\operatorname{div} a(z, D x(z))=\lambda f(z, x(z)) \quad \text { a.e. on } Z \\
\left.x\right|_{\partial Z}=0
\end{array}\right.
$$

Here, $a: Z \times \mathbb{R}^{N} \rightarrow \mathbb{R}^{N}$ is a Carathéodory map which is strictly monotone in the $y \in \mathbb{R}^{N}$ variable. The $p$-Laplacian is a particular case of the differential operator in $(P)_{\lambda}$, when $a(z, y) \equiv a(y)=|y|^{p-2} y$. However, in contrast to the $p$-Laplacian, the differential operator in $(P)_{\lambda}$ need not be homogeneous. Also, $f(z, x)$ is a Carathéodory nonlinearity, which near $\pm \infty$ exhibits a $(p-1)$-superlinear growth in the $x \in \mathbb{R}$ variable.

Presentado por / Submitted by Jesús Ildefonso Díaz Díaz.

Recibido / Received: 22 de diciembre de 2008. Aceptado / Accepted: 4 de marzo de 2009.

Palabras clave / Keywords: p-Laplacian-like operator; superlinear nonlinearity, strong deformation retract; critical groups and Morse theory.

Mathematics Subject Classifications: 35J25, 35J80, 58E05.

(C) 2009 Real Academia de Ciencias, España. 
Our goal is to prove existence and multiplicity theorems for problem $(P)_{\lambda}$, which are valid for all $\lambda>0$. In the past, problem $(P)_{\lambda}$ was investigated primarily in the context of equations driven by the Laplacian (semilinear problems), i.e., $a(z, y) \equiv a(y)=y$. The first such work was that by Ambrosetti-Rabinowitz [2] (see also Ambrosetti-Brezis-Cerami [1]), who employed the following condition on the nonlinearity $f(z, \cdot)$, known in the literature as the Ambrosetti-Rabinowitz condition (AR-condition, for short): there exist $\mu>2$ and $M>0$ such that

$$
0<\mu F(z, x) \leq f(z, x) x \quad \text { for all } z \in Z \text { and all }|x| \geq M
$$

where $f: \bar{Z} \times \mathbb{R} \rightarrow \mathbb{R}$ is a continuous function and $F(z, x)=\int_{0}^{x} f(z, s) \mathrm{d} s$.

A direct integration of (1), leads to the following weaker condition for the potential function $F(z, x)$,

$$
F(z, x) \geq \hat{c}_{1}|x|^{\mu}-\hat{c}_{2} \quad \text { for all } z \in \bar{Z} \text { and all } x \in \mathbb{R} \text {, with } \hat{c}_{1}, \hat{c}_{2}>0 .
$$

From (2), we infer that $F(z, \cdot)$ exhibits at least a $\mu$-polynomial growth near $\pm \infty$ and, since $\mu>p=2$, it also satisfies the much weaker condition

$$
\lim _{|x| \rightarrow \infty} \frac{F(z, x)}{x^{2}}=+\infty \quad \text { uniformly for a.a. } z \in Z \text {. }
$$

More recently, Schechter-Zou [28] considered problem $(P)_{\lambda}$ with the Laplacian differential operator (semilinear problem), but replaced the AR-condition with a unilateral version of (3), namely, they assumed that

$$
\lim _{x \rightarrow-\infty} \frac{F(z, x)}{x^{2}}=+\infty \quad \text { or } \quad \lim _{x \rightarrow+\infty} \frac{F(z, x)}{x^{2}}=+\infty \quad \text { uniformly for a.a. } z \in Z .
$$

Under (4), Schechter-Zou [28] proved that problem $(P)_{\lambda}$ (with the Laplace operator) has a nontrivial solution for almost all $\lambda>0$. Moreover, they proved the existence of a nontrivial solution for all $\lambda>0$, if (3) holds and one of the following two conditions is true:

$\left(\mathrm{C}_{1}\right) \mathcal{G}(z, x)=f(z, x) x-2 F(z, x)$ is convex in $x \in \mathbb{R}$, for all $z \in Z$;

$\left(\mathrm{C}_{2}\right)$ there exist constants $\hat{c}_{3}>0, \mu>2$ and $r \geq 0$ such that

$$
\mu F(z, x)-f(z, x) x \leq \hat{c}_{3}\left(1+x^{2}\right) \quad \text { for all } z \in Z \text {, all }|x| \geq r .
$$

Note that from condition $\left(\mathrm{C}_{2}\right)$, we have

$$
\begin{gathered}
\mu-\frac{\hat{c}_{3}\left(1+x^{2}\right)}{F(z, x)} \leq \frac{f(z, x) x}{F(z, x)} \quad \text { for all } z \in Z, \text { all }|x| \geq r, \\
\Downarrow \\
\mu \leq \liminf _{|x| \rightarrow \infty} \frac{f(z, x) x}{F(z, x)} \quad \text { uniformly for a.a. } z \in Z
\end{gathered}
$$

Therefore, condition $\left(\mathrm{C}_{2}\right)$ and the AR-condition (see (1)) are in fact equivalent.

On the other hand, condition $\left(\mathrm{C}_{1}\right)$ (i.e., the convexity of the function $x \mapsto \mathcal{G}(z, x)$ ) is actually stronger than the following one:

$\left(\mathrm{C}_{3}\right)$ there exists $M_{0}>0$ such that for all $z \in Z$

$$
x \mapsto \frac{f(z, x)}{x} \quad \text { is increasing on } x \geq M_{0} \text { and decreasing on } x \geq-M_{0} \text {. }
$$


A global version of $\left(\mathrm{C}_{3}\right)$ was used by Zhou [32], who studied nonparametric problems driven by the Laplacian.

Very recently, Miyagaki-Souto [19] used condition $\left(\mathrm{C}_{3}\right)$ to show that problem $(P)_{\lambda}$, with the Laplace differential operator, has a nontrivial solution for all $\lambda>0$.

Extensions to nonlinear parametric problems, driven by the $p$-Laplacian, were obtained by Guo [13], Guo-Webb [14], Hai [15], Motreanu-Motreanu-Papageorgiou [21], and Perera [27]. However, with the exception of [21], none of the aforementioned works deals with $(p-1)$-superlinear nonlinearities (i.e. nonlinearities which satisfy $\lim _{|x| \rightarrow \infty} \frac{f(z, x)}{|x|^{p-2} x}=+\infty$ uniformly for a.a. $z \in Z$ ) and they prove existence and multiplicity results, valid for all $\lambda>\lambda^{*}$, for some $\lambda^{*}>0$. In [21], the authors prove a multiplicity result (existence of three nontrivial solutions) for a nonlinearity $f(z, x)$ with general polynomial growth in $x \in \mathbb{R}$. However, their result is local in $\lambda>0$, namely, it is valid only for $\lambda \in\left(0, \lambda_{0}^{*}\right)$ and some $\lambda_{0}^{*}>0$. Finally, we should mention the recent work of Papageorgiou-Rocha-Staicu [25], where the authors prove multiplicity theorems for $p$-Laplacian equations with a $(p-1)$-superlinear nonlinearity $f(z, \cdot)$ and no parameter $\lambda>0$. They employ the AR-condition and in their multiplicity theorems, they provide information about the sign of all the solutions.

Our approach, in this work, combines variational methods with Morse theory and suitable truncation techniques. The structure of the work is the following. In the next Section, we present the background material necessary to follow the arguments in the subsequent sections. In Section 3, we prove an existence result (valid for all $\lambda>0$ ), using variational methods based on the critical point theory (mountain pass theorem). In Section 4, by combining variational arguments with suitable truncation techniques and Morse theory (in infinite dimensional Banach spaces), we prove a multiplicity theorem (existence of three nontrivial smooth solutions, two of which have opposite constant sign), which is valid for all $\lambda>0$. For the semilinear case, a similar result can be found in Perera [26], but his nonlinearity contains a negative concave term and so near the origin the behavior is different.

\section{Mathematical Background}

In the analysis of problem $(P)_{\lambda}$, we will use the Sobolev space $W_{0}^{1, p}(Z)$ and the Banach space

$$
C_{0}^{1}(\bar{Z})=\left\{u \in C^{1}(\bar{Z}):\left.u\right|_{\partial Z}=0\right\}
$$

We will also use the fact that the latter is an ordered Banach space, with order cone

$$
C_{+}=\left\{u \in C_{0}^{1}(\bar{Z}): u(z) \geq 0 \quad \text { for all } z \in \bar{Z}\right\} .
$$

This cone has a nonempty interior given by

$$
\operatorname{int} C_{+}=\left\{u \in C_{+}: u(z)>0 \text { for all } z \in Z \text { and } \frac{\partial u}{\partial n}(z)<0 \quad \text { for all } z \in \partial Z\right\},
$$

where $n(\cdot)$ denotes the outward unit normal on $\partial Z$.

Notation. Throughout this work, we denote the norm of $L^{p}(Z)$ by $\|\cdot\|_{p}$. For every $x \in W_{0}^{1, p}(Z)$, we set $\|x\|=\|D x\|_{p}$, and, for every $r \in \mathbb{R}, r^{ \pm}=\max \{ \pm r, 0\}$. The notation $\|\cdot\|$ will be also used to denote the $\mathbb{R}^{N}$-norm (besides its use as the norm of $W_{0}^{1, p}(Z)$ ), since it will be always clear from the context, which one are we referring to. We use $\rightarrow$ (resp. $\stackrel{w}{\longrightarrow}$ ) to denote strong convergence (resp. weak convergence).

\subsection{Critical point theory}

We start by recalling some basic notions and facts from critical point theory. Let $X$ be a Banach space and $X^{*}$ be its topological dual. By $<\cdot, \cdot>$ we denote the duality brackets for the pair $\left(X^{*}, X\right)$. Let $\varphi \in C^{1}(X)$. A point $x_{0} \in X$ is a critical point of $\varphi$ if $\varphi^{\prime}\left(x_{0}\right)=0$. A value $c \in \mathbb{R}$ is a critical value of $\varphi$ if exists a critical point $x_{0} \in X$ such that $\varphi\left(x_{0}\right)=c$. 
Definition 1 We define the following sets:

$$
\begin{array}{ll}
\varphi^{c}=\{x \in X: \varphi(x) \leq c\} & \text { (the sublevel set of } \varphi \text { at c), } \\
\dot{\varphi}^{c}=\{x \in X: \varphi(x)<c\} & \text { (the strict sublevel set of } \varphi \text { at c), } \\
K=\left\{x \in X: \varphi^{\prime}(x)=0\right\} & \text { (the critical set of } \varphi \text { ), }
\end{array}
$$

and

$$
K_{c}=\{x \in K: \varphi(x)=c\} \quad \text { (the critical set of } \varphi \text { at } c \text { ). }
$$

The following compactness notion will allow a minimax characterization of certain critical values of a $C^{1}$-functional.

Definition 2 We say that $\varphi \in C^{1}(X)$ satisfies the Cerami condition at level $c \in \mathbb{R}\left(C_{c^{-}}\right.$condition for short), if every sequence $\left\{x_{n}\right\}_{n \geq 1} \subseteq X$ satisfying

$$
\varphi\left(x_{n}\right) \rightarrow c \text { and }\left(1+\left\|x_{n}\right\|\right) \varphi^{\prime}\left(x_{n}\right) \rightarrow 0 \quad \text { in } X^{*} \text { as } n \rightarrow \infty,
$$

has a strongly convergent subsequence. We say that $\varphi$ satisfies the $C$-condition if it satisfies the $C_{c}$-condition for every level $c \in \mathbb{R}$.

This condition is a little more general than the usual Palais-Smale condition ( $P S$-condition for short). However, as it was shown by Bartolo-Benci-Fortunato [3], the deformation theorem and consequently the minimax theory of critical values of $\varphi \in C^{1}(X)$, is still valid if the $P S$-condition is replaced by the $C$-condition.

Theorem 1 ("Mountain pass theorem") If $X$ is a Banach space, $\varphi \in C^{1}(X), x_{0}, x_{1} \in X, r>0$, $\left\|x_{0}-x_{1}\right\|>r$,

$$
\begin{gathered}
\max \left\{\varphi\left(x_{0}\right), \varphi\left(x_{1}\right)\right\} \leq \inf \left\{\varphi(x):\left\|x-x_{0}\right\|=r\right\}=c_{0}, \\
\Gamma=\left\{\gamma \in C([0,1], X): \gamma(0)=x_{0} \text { and } \gamma(1)=x_{1}\right\} \\
c=\inf _{\gamma \in \Gamma} \max _{0 \leq t \leq 1} \varphi(\gamma(t))
\end{gathered}
$$

and $\varphi$ satisfies the $C_{c}$-condition, then $c \geq c_{0}$ and $c$ is a critical value of $\varphi$. Moreover, if $c=c_{0}$, then there exists a critical point $x \in X$ of $\varphi$ with critical value $c$ and $\|x\|=r$.

The notion of an operator of type $(S)_{+}$, introduced by Browder [5], will be useful in proving that a $C^{1}$-functional satisfies the $C$-condition (see Proposition 2).

Definition 3 A nonlinear map $A: X \rightarrow X^{*}$ is said to be of type $(S)_{+}$, if $x_{n} \stackrel{w}{\longrightarrow} x$ in $X$ and

$$
\limsup _{n \rightarrow \infty}<A\left(x_{n}\right), x_{n}-x>\leq 0,
$$

then $x_{n} \rightarrow x$ in $X^{*}$.

\subsection{Critical groups and Morse theory}

We now introduce some useful results from critical groups theory. If $\left(Y_{1}, Y_{2}\right)$ is a topological pair with $Y_{2} \subseteq Y_{1} \subseteq X$, then for every integer $k \geq 0$, we denote by $H_{k}\left(Y_{1}, Y_{2}\right)$ the $k^{\text {th }}$ relative singular homology group of the pair $\left(Y_{1}, Y_{2}\right)$, with integer coefficients.

Definition 4 The critical groups of $\varphi$ at an isolated critical point $x_{0} \in X$ with $\varphi\left(x_{0}\right)=c$, are defined by

$$
C_{k}\left(\varphi, x_{0}\right)=H_{k}\left(\varphi^{c} \cap \mathcal{U}, \varphi^{c} \cap \mathcal{U} \backslash\left\{x_{0}\right\}\right) \quad \text { for all } k \geq 0,
$$

where $\mathcal{U}$ is a neighborhood of $x_{0}$ such that $K \cap \varphi^{c} \cap \mathcal{U}=\left\{x_{0}\right\}$ (see Chang [6] and Mawhin-Willem [18]). 
The excision property of singular homology implies that the above definition is independent of the particular choice of neighborhood $\mathcal{U}$.

Suppose that $\varphi \in C^{1}(X)$ satisfies the $C$-condition and $\inf \varphi(K)>-\infty$. We choose $c<\inf \varphi(K)$.

Definition 5 The critical groups of $\varphi$ at infinity are defined by

$$
C_{k}(\varphi, \infty)=H_{k}\left(X, \varphi^{c}\right) \quad \text { for all } k \geq 0 \quad \text { (see Bartsch-Li [4]). }
$$

From the deformation theorem, we see that the above definition is independent of $c$. In fact, if $\eta<\inf \varphi(K)$, then

$$
C_{k}(\varphi, \infty)=H_{k}\left(X, \dot{\varphi}^{\eta}\right) \quad \text { for all } k \geq 0 .
$$

To see this, let $\vartheta<\eta<\inf \varphi(K)$. Then $\varphi^{\vartheta}$ is a strong deformation retract of $\dot{\varphi}^{\eta}$ (e.g., see Corvellec [7]). Hence,

$$
\begin{aligned}
& \begin{array}{c}
H_{k}\left(X, \varphi^{\vartheta}\right)=H_{k}\left(X, \dot{\varphi}^{\eta}\right) \quad \text { for all } k \geq 0, \\
\Downarrow
\end{array} \\
& C_{k}(\varphi, \infty)=H_{k}\left(X, \dot{\varphi}^{\eta}\right) \quad \text { for all } k \geq 0 \text {, }
\end{aligned}
$$

as claimed.

Definition 6 The Morse-type numbers of $\varphi$ at an isolated $x_{0} \in K$ are defined by

$$
m_{k}\left(x_{0}\right)=\operatorname{rank} C_{k}\left(\varphi, x_{0}\right) \quad \text { for all } k \geq 0,
$$

and the Morse-type polynomial by

$$
P\left(t, x_{0}\right)=\sum_{k \geq 0} m_{k}\left(x_{0}\right) t^{k}
$$

Definition 7 The Betti-type numbers of $\varphi$ are defined by

$$
\beta_{k}=\operatorname{rank} C_{k}(\varphi, \infty) \quad \text { for all } k \geq 0,
$$

and the Betti-type polynomial by

$$
P(t, \infty)=\sum_{k \geq 0} \beta_{k} t^{k}
$$

Suppose that $K$ is a finite set. Then, the Morse relation says that there is a polynomial $Q(t)$ with nonnegative integer coefficients such that

$$
\sum_{x \in K} P(t, x)=P(t, \infty)+(1+t) Q(t),
$$

(see Chang [6, p. 36], and Mawhin-Willem [18, p. 184]).

\subsection{The first eigenvalue of the negative Dirichlet $p$-Laplacian}

In what follows, $\lambda_{1}$ denotes the first eigenvalue of the negative Dirichlet $p$-Laplacian (which it is denoted by $\left(-\Delta_{p}, W_{0}^{1, p}(Z)\right)$ ). We know that $\lambda_{1}>0$ and it is simple (i.e., the corresponding eigenspace is one dimensional). Moreover, it admits the following characterization

$$
\lambda_{1}=\inf \left\{\frac{\|D u\|_{p}^{p}}{\|u\|_{p}^{p}}: u \in W_{0}^{1, p}(Z) \text { and } u \neq 0\right\} .
$$

The infimum in (6) is attained on the eigenspace of $\lambda_{1}$. Also, by $u_{1}$ we denote the $L^{p}$-normalized eigenfunction corresponding to $\lambda_{1}$. From (6), it is clear that $u_{1}$ does not change sign and so we may assume that $u_{1} \geq 0$. In fact, nonlinear regularity theory implies that $u_{1} \in C_{+} \backslash\{0\}$ and invoking the nonlinear strong maximum principle of Vazquez [30], we have $u_{1} \in \operatorname{int} C_{+}$. 


\section{Existence Theorems}

In this section, we examine the existence of nontrivial solutions for problem $(P)_{\lambda}$, with $\lambda>0$. Using variational methods and, in particular, the mountain pass theorem (see Theorem 1), we show that for every $\lambda>0$, problem $(P)_{\lambda}$ has a nontrivial bounded solution. Moreover, if we strengthen the conditions on the map $a(z, y)$, we show that the nontrivial solution is also smooth.

The hypotheses on the map $a(z, y)$ are the following:

$H(a): a(z, y)=\nabla_{y} G(z, y)$ where $G: Z \times \mathbb{R}^{N} \rightarrow \mathbb{R}$ is a function such that

(i) for all $y \in \mathbb{R}^{N}, z \mapsto G(z, y)$ is measurable;

(ii) for almost all $z \in Z, y \mapsto G(z, y)$ is $C^{1}$, strictly convex and $G(z, 0)=0$;

(iii) for almost all $z \in Z$ and all $y \in \mathbb{R}^{N}$, we have

$$
\|a(z, y)\|=\left\|\nabla_{y} G(z, y)\right\| \leq a_{0}(z)+c_{0}\|y\|^{p-1},
$$

with $a_{0} \in L^{\infty}(Z)_{+}, c_{0}>0$ and $1<p<\infty$;

(iv) for almost all $z \in Z$ and all $y \in \mathbb{R}^{N}$, we have

$$
(a(z, y), y)_{\mathbb{R}^{N}} \leq p G(z, y)
$$

(v) there exists $\hat{c}>0$ such that for almost all $z \in Z$ and all $y \in \mathbb{R}^{N}$, we have

$$
\hat{c}\|y\|^{p} \leq p G(z, y)
$$

Remark 1 The above hypotheses on a $(z, y)$ are considerably more general than those employed by De Napoli-Mariani [9], who also deal with equations driven by p-Laplace-like operators. In [9], $G \in C^{1}\left(\bar{Z} \times \mathbb{R}^{N}\right)$ and it is assumed that for all $z \in \bar{Z}, G(z, \cdot)$ is strongly convex. This condition, in the particular case of the $p$-Laplacian (i.e., when $G(y)=\frac{1}{p}\|y\|^{p}$ ), implies that $p \geq 2$. No such restriction is necessary here.

In what follows, we present some characteristic examples of functions $G(z, y)$ with $a(z, y)=\nabla_{y} G(z, y)$ satisfying the hypotheses $H(a)$.

Example 1 Let $G(z, y) \equiv G(y)=\frac{1}{p}\|y\|^{p}$ with $1<p<\infty$. In this case, $a(y)=\nabla G(y)=\|y\|^{p-2} y$. The corresponding differential operator is the p-Laplacian

$$
\Delta_{p} u=\operatorname{div}\left(\|D u\|^{p-2} D u\right) .
$$

Example 2 Let $G(z, y)=\frac{1}{p} a_{1}(z)\|y\|^{p}+\frac{1}{r} a_{2}(z)\|y\|^{r}$ with $a_{1}, a_{2} \in L^{\infty}(Z)_{+}, a_{1}(z) \geq c_{0}>0$ for a.a. $z \in Z$ and $1<r<p<\infty$. Then

$$
a(z, y)=a_{1}(z)\|y\|^{p-2} y+a_{2}(z)\|y\|^{r-2} y
$$

and the corresponding differential operator is the weighted $(p, r)$-Laplacian

$$
a_{1}(z) \Delta_{p} u+a_{2}(z) \Delta_{r} u
$$

Example 3 Let $G(z, y)=\frac{1}{p} a_{1}(z)\|y\|^{p}+\frac{1}{r} \ln \left(1+\|y\|^{r}\right)$, with $a_{1} \in L^{\infty}(Z)_{+}, a_{1}(z) \geq c_{0}>0$ for a.a. $z \in Z$ and $1<r \leq p<\infty$. Then

$$
a(z, y)=a_{1}(z)\|y\|^{p-2} y+\|y\|^{r-2} y \frac{1}{1+\|y\|^{r}} .
$$


Example 4 Let $G(z, y) \equiv G(y)=\frac{1}{p}\left[\left(1+\|y\|^{2}\right)^{\frac{p}{2}}-1\right]$ with $1<p<\infty$. Then

$$
a(y)=\left(1+\|y\|^{2}\right)^{\frac{p-2}{2}} y .
$$

The corresponding differential operator is the generalized mean curvature operator

$$
\operatorname{div}\left(\left(1+\|D u\|^{2}\right)^{\frac{p-2}{2}} D u\right)
$$

\section{Example 5 Let}

$$
G(z, y) \equiv G(y)= \begin{cases}\frac{1}{r}\|y\|^{r} & \text { if }\|y\| \leq 1 \\ \frac{1}{p}\|y\|^{p}+\frac{p-r}{p r} & \text { if }\|y\|>1\end{cases}
$$

with $1<r<p<\infty$. Then

$$
a(y)= \begin{cases}\|y\|^{r-2} y & \text { if }\|y\| \leq 1 \\ \|y\|^{p-2} y & \text { if }\|y\|>1\end{cases}
$$

Example 6 Let $G(z, y)=\frac{1}{2}(A(z) y, y)_{\mathbb{R}^{N}}$, with $A \in L^{\infty}\left(Z, \mathbb{R}^{N \times N}\right)$ and $A(z) \geq c_{0} I_{N \times N}$ for a.a $z \in Z$, where $c_{0}>0$ and $I_{N \times N}$ is the $(N \times N)$-identity matrix.

Definition 8 Let $S: W_{0}^{1, p}(Z) \rightarrow W^{-1, p^{\prime}}(Z)$, with $\frac{1}{p}+\frac{1}{p^{\prime}}=1$, be the nonlinear operator defined by

$$
<S(x), y>=\int_{Z}(a(z, D x), D y)_{\mathbb{R}^{N}} \mathrm{~d} z \quad \text { for all } x, y \in W_{0}^{1, p}(Z) .
$$

From Papageorgiou-Rocha-Staicu [24, Proposition 2], we have:

Proposition 1 If hypotheses $H(a)$ hold, then the operator $S: W_{0}^{1, p}(Z) \rightarrow W^{-1, p^{\prime}}(Z)$ defined by (7) is maximal monotone, strictly monotone, and of type $(S)_{+}$.

The hypotheses on the nonlinearity $f(z, x)$ are the following:

$H_{1}(f): f: Z \times \mathbb{R} \rightarrow \mathbb{R}$ is a function such that $f(z, 0)=0$ a.e. on $Z$ and

(i) for all $x \in \mathbb{R}, z \mapsto f(z, x)$ is measurable;

(ii) for almost all $z \in Z, x \mapsto f(z, x)$ is continuous;

(iii) for almost all $z \in Z$ and all $x \in \mathbb{R}$, we have

$$
|f(z, x)| \leq a(z)+c|x|^{r-1}
$$

with $a \in L^{\infty}(Z)_{+}, c>0$ and $p<r<p^{*}$;

(iv) if $F(z, x)=\int_{0}^{x} f(z, s) \mathrm{d} s$, then

$$
\lim _{|x| \rightarrow \infty} \frac{F(z, x)}{|x|^{p}}=+\infty \quad \text { uniformly for a.a. } z \in Z
$$

and there exists $\tau \geq 1, \tau \in(\theta, r)$ with $\theta=(r-p) \max \left\{\frac{N}{p}, 1\right\}$, such that

$$
\liminf _{|x| \rightarrow \infty} \frac{f(z, x) x-p F(z, x)}{|x|^{\tau}}>0 \quad \text { uniformly for a.a. } z \in Z \text {; }
$$


(v) we have

$$
\lim _{x \rightarrow 0} \frac{f(z, x)}{|x|^{p-2} x}=0 \quad \text { uniformly for a.a. } z \in Z .
$$

As usual, $p^{*}$ denotes the Sobolev critical exponent, i.e., $p^{*}=\frac{N p}{N-p}$ if $p<N$ and $p^{*}=+\infty$ if $p \geq N$.

Remark 2 Hypothesis $H_{1}(f)(\mathrm{iv})$ is a p-superlinearity condition on the potential $F(z, \cdot)$. Evidently, if $\lim _{|x| \rightarrow \infty} \frac{f(z, x)}{|x|^{p-2} x}=+\infty$ uniformly for a.a. $z \in Z$, then $\lim _{|x| \rightarrow \infty} \frac{F(z, x)}{|x|^{p}}=+\infty$ uniformly for a.a. $z \in Z$. The second part of the hypothesis $H_{1}(f)(\mathrm{iv})$ replaces the AR-condition (see (1)). It was first used by CostaMagalhaes [8].

Example 7 Consider the following potential function $F(z, x) \equiv F(x)$ (for the sake of simplicity, we drop the dependence of $F$ on the $z$ variable)

$$
F(x)=\frac{1}{p}|x|^{p} \ln (1+|x|)+\cos \left(|x|^{p}\right)-1 .
$$

Then

$$
f(x)=|x|^{p-2} x\left(\ln (1+|x|)+\frac{|x|}{p(1+|x|)}\right)-p|x|^{p-2} x \sin \left(|x|^{p}\right) .
$$

Let $\epsilon>0$ be such that $N \epsilon<p^{2}$. If $\mu=p$ and $r=p+\epsilon$, then all hypotheses $H_{1}(f)$ are satisfied. However, the AR-condition (see (1)) is not satisfied and since

$$
\frac{f(x)}{|x|^{p-2} x}=\ln (1+|x|)+\frac{|x|}{p(1+|x|)}-p \sin \left(|x|^{p}\right),
$$

then, when $p=2$, condition $\left(\mathrm{C}_{3}\right)$ is not satisfied and so the semilinear existence result of MiyagakiSouto [9] does not apply on this nonlinearity.

Definition 9 Let $\lambda>0$ and $\varphi_{\lambda}: W_{0}^{1, p}(Z) \rightarrow \mathbb{R}$ be the Euler functional for problem $(P)_{\lambda}$ defined by

$$
\varphi_{\lambda}(x)=\int_{Z} G(z, D x(z)) \mathrm{d} z-\lambda \int_{Z} F(z, x(z)) \mathrm{d} z \quad \text { for all } x \in W_{0}^{1, p}(Z) .
$$

Evidently, $\varphi_{\lambda} \in C^{1}\left(W_{0}^{1, p}(Z)\right)$ and, for all $x \in W_{0}^{1, p}(Z)$, we have

$$
\varphi_{\lambda}^{\prime}(x)=S(x)-\lambda N(x),
$$

where $N(x)(\cdot)=f(\cdot, x(\cdot))$ and $S$ is the operator defined in (7) (see also Ubilla [29]).

Proposition 2 If $\lambda>0$, hypotheses $H(a)$ and $H_{1}(f)$ hold, then $\varphi_{\lambda}$ satisfies the $C$-condition.

PROOF. We consider a sequence $\left\{x_{n}\right\}_{n \geq 1} \subseteq W_{0}^{1, p}(Z)$ such that

$$
\left|\varphi_{\lambda}\left(x_{n}\right)\right| \leq M_{1} \quad \text { for some } M_{1}>0, \quad \text { all } n \geq 1,
$$

and

$$
\left(1+\left\|x_{n}\right\|\right) \varphi_{\lambda}^{\prime}\left(x_{n}\right) \rightarrow 0 \quad \text { in } W_{0}^{1, p}(Z) \quad \text { as } n \rightarrow \infty .
$$

Claim. The sequence $\left\{x_{n}\right\}_{n \geq 1} \subseteq W_{0}^{1, p}(Z)$ is bounded.

We argue by contradiction. So, suppose that the Claim is not true. By passing to a suitable subsequence, if necessary, we may assume that $\left\|x_{n}\right\| \rightarrow \infty$. From (10), for all $v \in W_{0}^{1, p}(Z)$, we have

$$
\begin{gathered}
\left|<\varphi_{\lambda}^{\prime}\left(x_{n}\right), v>\right| \leq \frac{\epsilon_{n}}{1+\left\|x_{n}\right\|}\|v\| \quad \text { with } \epsilon_{n} \downarrow 0, \\
\Downarrow \\
\left|<S\left(x_{n}\right), v>-\lambda \int_{Z} f\left(z, x_{n}\right) v \mathrm{~d} z\right| \leq \frac{\epsilon_{n}}{1+\left\|x_{n}\right\|}\|v\| \quad \text { with } \epsilon_{n} \downarrow 0 .
\end{gathered}
$$


In (11), we choose $v=x_{n} \in W_{0}^{1, p}(Z)$, then

$$
-\int_{Z}\left(a\left(z, D x_{n}\right), D x_{n}\right)_{\mathbb{R}^{N}} \mathrm{~d} z+\lambda \int_{Z} f\left(z, x_{n}\right) x_{n} \mathrm{~d} z \leq \epsilon_{n} \quad \text { for all } n \geq 1 .
$$

On the other hand, from (9), we have

$$
\int_{Z} p G\left(z, x_{n}\right) \mathrm{d} z-\lambda \int_{Z} p F\left(z, x_{n}\right) \mathrm{d} z \leq p M_{1} \quad \text { for all } n \geq 1 .
$$

We add (12) and (13), obtaining

$$
\int_{Z}\left[p G\left(z, D x_{n}\right)-\left(a\left(z, D x_{n}\right), D x_{n}\right)_{\mathbb{R}^{N}}\right] \mathrm{d} z+\lambda \int_{Z}\left[f\left(z, x_{n}\right) x_{n}-p F\left(z, x_{n}\right)\right] \mathrm{d} z \leq M_{2}
$$

for some $M_{2}>0$ and all $n \geq 1$. By virtue of hypothesis $H(a)$ (iv), we have

$$
\int_{Z}\left[p G\left(z, D x_{n}\right)-\left(a\left(z, D x_{n}\right), D x_{n}\right)_{\mathbb{R}^{N}}\right] \mathrm{d} z \geq 0 \quad \text { for all } n \geq 1 \text {. }
$$

Hypothesis $H_{1}(f)\left(\right.$ iv) implies that we can find $\beta>0$ and $M_{3} \equiv M_{3}(\beta)>0$ such that

$$
\beta|x|^{\tau} \leq f(z, x) x-p F(z, x) \quad \text { for a.a. } z \in Z \text {, all }|x| \geq M_{3} .
$$

In addition, hypothesis $H_{1}(f)$ (iii) implies the existence of a $M_{4}>0$ such that

$$
|f(z, x) x-p F(z, x)| \leq M_{4} \quad \text { for a.a. } z \in Z \text {, all }|x|<M_{3} .
$$

Combining (16) and (17), we infer that there exists $c_{1}>0$ such that

$$
\beta|x|^{\tau}-c_{1} \leq f(z, x) x-p F(z, x) \quad \text { for a.a. } z \in Z \text {, all } x \in \mathbb{R} .
$$

We return to (14) and use (15) and (18). Then

$$
\begin{gathered}
\beta \int_{Z}\left|x_{n}\right|^{\tau} \mathrm{d} z \leq M_{5} \quad \text { for some } M_{5}>0, \text { all } n \geq 1, \\
\Downarrow \\
\left\{x_{n}\right\}_{n \geq 1} \subseteq L^{\tau}(Z) \quad \text { is bounded. }
\end{gathered}
$$

Recall that $\tau \leq r<p^{*}$ (see hypothesis $H_{1}(f)$ (iv)). So, we can find $t \in[0,1)$ such that

$$
\frac{1}{r}=\frac{1-t}{\tau}+\frac{t}{p^{*}}
$$

Invoking the interpolation inequality (e.g., see Gasinski-Papageorgiou [11, p. 905]), we have

$$
\left\|x_{n}\right\|_{r} \leq\left\|x_{n}\right\|_{\tau}^{1-t}\left\|x_{n}\right\|_{p^{*}}^{t}
$$

which implies

$$
\left\|x_{n}\right\|_{r}^{r} \leq M_{6}\left\|x_{n}\right\|_{p^{*}}^{t r} \quad \text { for some } M_{6}>0, \text { all } n \geq 1
$$

From (11) with $v=x_{n} \in W_{0}^{1, p}(Z)$, we have

$$
\int_{Z}\left(a\left(z, D x_{n}\right), D x_{n}\right)_{\mathbb{R}^{N}} \mathrm{~d} z-\lambda \int_{Z} f\left(z, x_{n}\right) x_{n} \mathrm{~d} z \leq \epsilon_{n} .
$$


Since for a.a. $z \in Z, G(z, \cdot)$ is convex (see hypothesis $H(a)($ ii)), we have

$$
(a(z, y),-y)_{\mathbb{R}^{N}} \leq G(z, 0)-G(z, y)=-G(z, y) \quad \text { for a.a. } z \in Z \text {, all } y \in \mathbb{R}^{N},
$$

which implies that

$$
\frac{\hat{c}}{p}\|y\|^{p} \leq G(z, y) \leq(a(z, y), y)_{\mathbb{R}^{N}} \quad \text { for a.a. } z \in Z \text {, all } y \in \mathbb{R}^{N}
$$

(see hypothesis $H(a)(\mathrm{v})$ ). On the other hand, hypotheses $H_{1}(f)(\mathrm{iii})$ and (v) imply that, given $\epsilon>0$, we can find $c_{\epsilon}>0$ such that

$$
|f(z, x) x| \leq \epsilon|x|^{p}+c_{\epsilon}|x|^{r} \quad \text { for a.a. } z \in Z \text {, all } x \in \mathbb{R} .
$$

We return to (21) and use (22) and (23), then

$$
\begin{aligned}
\frac{\hat{c}}{p}\left\|D x_{n}\right\|_{p}^{p} & \leq \epsilon_{n}+\lambda \epsilon\left\|x_{n}\right\|_{p}^{p}+\lambda c_{\epsilon}\left\|x_{n}\right\|_{r}^{r} \\
& \leq \epsilon_{n}+\lambda\left(\epsilon\left\|x_{n}\right\|_{p}^{p}+c_{3}\left\|x_{n}\right\|_{p^{*}}^{t r}\right)
\end{aligned}
$$

for some $c_{3} \equiv c_{3}(\epsilon)>0$, and all $n \geq 1$ (see (20)).

Let $u_{n}=\frac{x_{n}}{\left\|x_{n}\right\|}, n \geq 1$. Then, $\left\|u_{n}\right\|=1$ for all $n \geq 1$ and so, we may assume that

$$
u_{n} \stackrel{w}{\longrightarrow} u \text { in } W_{0}^{1, p}(Z) \quad \text { and } \quad u_{n} \rightarrow u \text { in } L^{p}(Z) \quad \text { as } n \rightarrow \infty .
$$

From (24), we have

$$
\frac{\hat{c}}{p}\left\|D u_{n}\right\|_{p}^{p} \leq \frac{\epsilon_{n}}{\left\|x_{n}\right\|^{p}}+\lambda\left(\epsilon\left\|u_{n}\right\|_{p}^{p}+\frac{c_{4}}{\left\|x_{n}\right\|^{p-t r}}\left\|u_{n}\right\|^{t r}\right)
$$

for some $c_{4}>0$, and $n \geq 1$. The hypothesis on $\tau$ (see hypothesis $H_{1}(f)$ (iv)) implies that $t r<p$. So, if in (26) we pass to the limit as $n \rightarrow \infty$, we obtain

$$
\frac{\hat{c}}{p}\|D u\|_{p}^{p} \leq \lambda \epsilon\|u\|_{p}^{p} \leq \frac{\lambda}{\lambda_{1}} \epsilon\|D u\|_{p}^{p} \leq \frac{\lambda}{\lambda_{1}} \epsilon,
$$

(see (6) and (25)). But $\epsilon>0$ was arbitrary, so we let $\epsilon \downarrow 0$ and obtain $u=0$. Then, from (24), we have $\left\|u_{n}\right\| \rightarrow 0$, a contradiction to the fact that $\left\|u_{n}\right\|=1$ for all $n \geq 1$. This proves the Claim.

Because of the Claim, we may assume that

$$
x_{n} \stackrel{w}{\longrightarrow} x \quad \text { in } W_{0}^{1, p}(Z) \quad \text { and } \quad x_{n} \rightarrow x \quad \text { in } L^{r}(Z) .
$$

From (11), with $v=x_{n}-x \in W_{0}^{1, p}(Z)$, we have

$$
\left|<S\left(x_{n}\right), x_{n}-x>-\lambda \int_{Z} f\left(z, x_{n}\right)\left(x_{n}-x\right) \mathrm{d} z\right| \leq \frac{\epsilon_{n}}{1+\left\|x_{n}\right\|}\left\|x_{n}-x\right\| .
$$

Evidently,

$$
\int_{Z} f\left(z, x_{n}\right)\left(x_{n}-x\right) \mathrm{d} z \rightarrow 0 \quad \text { as } n \rightarrow \infty \quad \text { (see } H_{1}(f) \text { (iii) and (27)). }
$$

Therefore, if in (22) we pass to the limit as $n \rightarrow \infty$, then

$$
\lim _{n \rightarrow \infty}<S\left(x_{n}\right), x_{n}-x>=0,
$$

which implies that

$$
x_{n} \rightarrow x \quad \text { in } W_{0}^{1, p}(Z) \quad \text { as } n \rightarrow \infty \quad \text { (see Proposition 1). }
$$

We conclude that $\varphi_{\lambda}$ satisfies the $C$-condition for all $\lambda>0$. 
Proposition 3 If $\lambda>0$, hypotheses $H(a)$ and $H_{1}(f)$ hold, then $x=0$ is a local minimizer of $\varphi_{\lambda}$. Proof. By virtue of hypothesis $H_{1}(f)(\mathrm{v})$, given $\epsilon>0$, we can find $\delta \equiv \delta(\epsilon)>0$ such that

$$
f(z, x) \leq \frac{\epsilon}{\lambda} x^{p-1} \quad \text { for a.a. } z \in Z \text { and all } x \in[0, \delta]
$$

and

$$
f(z, x) \geq \frac{\epsilon}{\lambda}|x|^{p-2} x \quad \text { for a.a. } z \in Z \text { and all } x \in[-\delta, 0] .
$$

From (29) and (30), after integration, we obtain

$$
F(z, x) \leq \frac{\epsilon}{\lambda p}|x|^{p} \quad \text { for a.a. } z \in Z \text { and all }|x| \leq \delta .
$$

This, combined with hypothesis $H_{1}(f)\left(\right.$ iii), implies that we can find $c_{5}>0$ such that

$$
F(z, x) \leq \frac{\epsilon}{\lambda p}|x|^{p}+c_{5}|x|^{r} \quad \text { for a.a. } z \in Z \text { and all } x \in \mathbb{R} .
$$

Hence, for all $x \in W_{0}^{1, p}(Z)$ and some $c_{6}>0$, we have

$$
\begin{aligned}
\varphi_{\lambda}(x) & =\int_{Z} G(z, D x) \mathrm{d} z-\lambda \int_{Z} F(z, x) \mathrm{d} z \\
& \geq \frac{\hat{c}}{p}\|D x\|_{p}^{p}-\frac{\epsilon}{p}\|x\|_{p}^{p}-\lambda c_{6}\|D x\|_{p}^{r} \\
& \geq \frac{1}{p}\left(\hat{c}-\frac{\epsilon}{\lambda_{1}}\right)\|D x\|_{p}^{p}-\lambda c_{6}\|D x\|_{p}^{r} \quad \text { (see } H(a)(\mathrm{v}) \text { and (31)) }
\end{aligned}
$$

Choose $\epsilon \in\left(0, \lambda_{1} \hat{c}\right)$. Then, from (32) and since $r>p$, we see that we can find $\rho \in(0,1)$ small such that

$$
\varphi_{\lambda}(x)>0 \quad \text { for all } x \in W_{0}^{1, p}(Z) \text { with } 0<|x| \leq \rho,
$$

which implies that $x=0$ is a (strict) local minimizer of $\varphi_{\lambda}$.

As in Motreanu-Motreanu-Papageorgiou [22] (see the proof of Proposition 6), we can find $\rho_{\lambda}>0$ small such that

$$
0=\varphi_{\lambda}(0)<\inf \left\{\varphi_{\lambda}(u):\|u\|=\rho_{\lambda}\right\}=c_{\lambda} .
$$

Proposition 4 If $\lambda>0$, hypotheses $H(a)$ and $H_{1}(f)$ hold, then

$$
\varphi_{\lambda}\left(t u_{1}\right) \rightarrow-\infty \quad \text { as }|t| \rightarrow \infty
$$

where $u_{1} \in \operatorname{int} C_{+}$is the $L^{p}$-normalized principal eigenfunction of $\left(-\Delta_{p}, W_{0}^{1, p}(Z)\right.$ ) (see section 2.3).

Proof. From hypotheses $H_{1}(f)$ (iii), (iv), we see that given any $\eta>0$, we can find $c_{\eta}>0$ such that

$$
F(z, x) \geq \frac{\eta}{p}|x|^{p}-c_{\eta} \quad \text { for a.a. } z \in Z \text { and all } x \in \mathbb{R} .
$$

Hence, using the facts that $\lambda_{1}\left\|u_{1}\right\|_{p}^{p}=\left\|D u_{1}\right\|_{p}^{p}$ and $\left\|u_{1}\right\|_{p}=1$,

$$
\begin{aligned}
\varphi_{\lambda}\left(t u_{1}\right) & =\int_{Z} G\left(z, t u_{1}\right) \mathrm{d} z-\lambda \int_{Z} F\left(z, t u_{1}\right) \mathrm{d} z \\
& \leq \frac{|t|^{p}}{p}\left(c \lambda_{1}-\eta\right)+\hat{c}_{\eta} \quad \text { for some } c, \hat{c}_{\eta}>0 \quad \text { (see (34)). }
\end{aligned}
$$

Since $\eta>0$ was arbitrary, we may choose $\eta>c \lambda_{1}$. Then from (35), we conclude that $\varphi_{\lambda}\left(t u_{1}\right) \rightarrow-\infty$ as $|t| \rightarrow \infty$.

We are now ready for the existence theorem for problem $(P)_{\lambda}$. We show that it has a nontrivial bounded solution for every $\lambda>0$. 
Theorem 2 If hypotheses $H(a)$ and $H_{1}(f)$ hold, then, for every $\lambda>0$, problem $(P)_{\lambda}$ has a nontrivial solution $x_{0} \in W_{0}^{1, p}(Z) \cap L^{\infty}(Z)$.

Proof. Propositions 2, 4 and (33) permit the application of Theorem 1 (the mountain pass theorem). So, we obtain $x_{0} \in W_{0}^{1, p}(Z)$ such that

$$
\begin{gathered}
\varphi_{\lambda}(0)=0<c_{\lambda} \leq \varphi_{\lambda}\left(x_{0}\right) \\
\text { and } \varphi_{\lambda}^{\prime}\left(x_{0}\right)=0 .
\end{gathered}
$$

From (36), it follows that $x_{0} \neq 0$. From (37), we have $S\left(x_{0}\right)=\lambda N\left(x_{0}\right)$, which implies

$$
\left\{\begin{array}{l}
-\operatorname{div} a\left(z, D x_{0}(z)\right)=\lambda f\left(z, x_{0}(z)\right) \quad \text { a.e. on } Z \\
\left.x_{0}\right|_{\partial Z}=0
\end{array}\right.
$$

Theorem 7.1, p. 286, of Ladyzhenskaya-Uraltseva [16] implies that $x_{0} \in L^{\infty}(Z)$.

If we strengthen the conditions on the map $a(z, y)$, we can have more regularity for the solution $x_{0}$. The new, stronger hypotheses on the map $a(z, y)$ are the following:

$H_{1}(a): a(z, y)=\nabla_{y} G(z, y)$, where $G \in C^{1}\left(\bar{Z} \times \mathbb{R}^{N}\right) \cap C^{2}\left(\bar{Z} \times\left(\mathbb{R}^{N} \backslash\{0\}\right)\right)$, for all $z \in \bar{Z}$, we have $G(z, 0)=0, G(z, \cdot)$ is strictly convex and

(i) for all $(z, y) \in \bar{Z} \times\left(\mathbb{R}^{N} \backslash\{0\}\right)$, we have

$$
\left\|\nabla_{y} a(z, y)\right\| \leq c_{7}\|y\|^{p-2}
$$

for some $c_{7}>0$

(ii) for all $(z, y) \in \bar{Z} \times\left(\mathbb{R}^{N} \backslash\{0\}\right)$ and all $\xi \in \mathbb{R}^{N}$, we have

$$
c_{8}\|y\|^{p-2}\|\xi\|^{2} \leq\left(\nabla_{y} a(z, y) \xi, \xi\right)_{\mathbb{R}^{N}}
$$

for some $c_{8}>0$;

(iii) for all $(z, y) \in \bar{Z} \times \mathbb{R}^{N}$, we have

$$
(a(z, y), y)_{\mathbb{R}^{N}} \leq p G(z, y) .
$$

Remark 3 From hypothesis $H_{1}(a)(i)$, it follows that

$$
\|a(z, y)\| \leq \frac{c_{7}}{p-1}\|y\|^{p-1}
$$

for all $(z, y) \in \bar{Z} \times \mathbb{R}^{N}$ (compare with $H(a)\left(\right.$ iii)). Also, from hypothesis $H_{1}(a)(i i)$, we have

$$
\begin{gathered}
\frac{c_{8}}{p-1}\|y\|^{p-1} \leq(a(z, y), y)_{\mathbb{R}^{N}} \quad \text { for all }(z, x) \in \bar{Z} \times \mathbb{R}^{N}, \\
\Downarrow \\
\frac{c_{8}}{p(p-1)}\|y\|^{p} \leq G(z, y) \quad \text { for all }(z, y) \in \bar{Z} \times \mathbb{R}^{N},
\end{gathered}
$$

(compare with $H(a)(v)$ ). 
Example 8 The Example 1 not only satisfies $H(a)$ but also $H_{1}(a)$. The following functions $G(z, y)$, similar to Examples 2-6, with a $(z, y)=\nabla_{y} G(z, y)$ satisfy the new hypotheses $H_{1}(a)$ :

(1) $G(z, y)=\frac{1}{p} a_{1}(z)\|y\|^{p}+\frac{1}{r} a_{2}(z)\|y\|^{r}$, with $a_{1}, a_{2} \in C^{1}(\bar{Z}), a_{1}(z) \geq \hat{c}_{0}>0$ for all $z \in \bar{Z}$ and $1<r<p<\infty$;

(2) $G(z, y)=\frac{1}{p} a_{1}(z)\|y\|^{p}+\frac{1}{r} \ln \left(1+\|y\|^{r}\right)$, with $a_{1} \in C^{1}(\bar{Z}), a_{1}(z) \geq \hat{c}_{0}>0$ for all $z \in \bar{Z}$ and $1<r<p \stackrel{p}{<} \infty$;

(3) $G(y)=\frac{1}{p}\left(\left(1+\|y\|^{2}\right)^{\frac{p}{2}}-1\right)$, with $1<p<2$ (see Example 4);

(4) $G(z, y)=\frac{1}{2}(A(z) y, y)_{\mathbb{R}^{N}}$ with $A \in C^{1}\left(\bar{Z}, \mathbb{R}^{N \times N}\right)$ and $A(z)>0$ for all $z \in \bar{Z}$.

From Theorem 2 and Theorem 1 of Lieberman [17] (nonlinear regularity), we have:

Theorem 3 If hypotheses $H_{1}(a)$ and $H_{1}(f)$ hold, then, for every $\lambda>0$, problem $(P)_{\lambda}$ has a nontrivial solution $x_{0} \in C_{0}^{1}(\bar{Z})$.

\section{Multiple Nontrivial Solutions}

In this section, by strengthening further the conditions on $a(z, y)$ and $f(z, x)$, we prove a multiplicity result for problem $(P)_{\lambda}$ valid for all $\lambda>0$. To the best of our knowledge, we can not find in the literature such a multiplicity result, even when the differential operator is the Laplacian (semilinear parametric problem).

The new hypotheses on the map $a(z, y)$ are the following:

$H_{2}(a): a(z, y)=h(z,\|y\|) y$ for all $(z, y) \in \bar{Z} \times \mathbb{R}^{N}$, with $h(z, 0) \geq 0, h(z, t)>0$ for all $z \in \bar{Z}$ and all $t>0$, and

(i) $a \in C\left(\bar{Z} \times \mathbb{R}^{N}, \mathbb{R}^{N}\right) \cap C^{1}\left(\bar{Z} \times\left(\mathbb{R}^{N} \backslash\{0\}\right), \mathbb{R}^{N}\right)$;

(ii) for every $(z, y) \in \bar{Z} \times\left(\mathbb{R}^{N} \backslash\{0\}\right)$, we have

$$
\left\|\nabla_{y} a(z, y)\right\| \leq c_{9}\|y\|^{p-2}
$$

with $1<p<\infty$ and $c_{9}>0$;

(iii) for every $(z, y) \in \bar{Z} \times\left(\mathbb{R}^{N} \backslash\{0\}\right)$ and every $\xi \in \mathbb{R}^{N}$, we have

$$
c_{10}\|y\|^{p-2}\|\xi\|^{2} \leq\left(\nabla_{y} a(z, y) \xi, \xi\right)_{\mathbb{R}^{N}}
$$

for some $c_{10}>0$.

Remark 4 Set $g(z, t)=h(z, t)$ for all $(z, t) \in \bar{Z} \times \mathbb{R}_{+}$, where $R_{+}=[0, \infty)$. From hypotheses $H_{2}(a)$ above, we have the following one-dimensional estimate

$$
c_{10} t^{p-2} \leq \frac{\partial}{\partial t} g(z, t) \leq c_{9} t^{p-2}
$$

for all $z \in \bar{Z}$ and all $t>0$, which implies that the map $t \mapsto g(z, t)$ is strictly increasing on $(0,+\infty)$ for all $z \in \bar{Z}$. We set $G_{0}(z, t)=\int_{0}^{t} g(z, s) \mathrm{d} s$ for all $(z, t) \in \bar{Z} \times \mathbb{R}_{+}$. Then,

$$
\frac{\partial}{\partial t} G_{0}(z, t)=g(z, t) \quad \text { for all } z \in \bar{Z} \text { and } t \geq 0 .
$$


It follows that for all $z \in \bar{Z}$, the function $t \mapsto G_{0}(z, t)$ is strictly convex and strictly increasing on $\mathbb{R}_{+}$. We set

$$
G(z, y)=G_{0}(z,\|y\|) \quad \text { for all }(z, y) \in \bar{Z} \times \mathbb{R}^{N} .
$$

Evidently, for all $z \in \bar{Z}, G(z, \cdot)$ is convex. Moreover, for all $(z, y) \in \bar{Z} \times\left(\mathbb{R}^{N} \backslash\{0\}\right)$, we have

$$
\begin{aligned}
\nabla_{y} G(z, y) & =\frac{\partial}{\partial t} G_{0}(z,\|y\|) \frac{y}{\|y\|} & & \\
& =g(z,\|y\|) \frac{y}{\|y\|} & & (\text { see }(38)) \\
& =h(z,\|y\|) y & & (\text { since } g(z, t)=h(z, t) t) \\
& =a(z, y) . & &
\end{aligned}
$$

Using the hypotheses $H_{2}(a)$, we can easily prove the following Lemma.

Lemma 1 If hypotheses $H_{2}(a)$ hold, then:

(a) for all $z \in \bar{Z}, y \mapsto a(z, y)$ is maximal monotone and strictly monotone;

(b) for all $(z, y) \in \bar{Z} \times \mathbb{R}^{N}$, $\|a(z, y)\| \leq \frac{c_{9}}{p-1}\|y\|^{p-1}$;

(c) for all $(z, y) \in \bar{Z} \times \mathbb{R}^{N}, \frac{c_{10}}{p-1}\|y\|^{p} \leq(a(z, y), y)_{\mathbb{R}^{N}}$.

A straightforward consequence of the above Lemma, is the following result:

Lemma 2 If hypotheses $H_{2}(a)$ hold, then we have

$$
\frac{c_{10}}{p(p-1)}\|y\|^{p} \leq G(z, y) \leq \frac{c_{9}}{p(p-1)}\|y\|^{p} \quad \text { for all }(z, y) \in \bar{Z} \times \mathbb{R}^{N} .
$$

Example 9 The following are examples of maps $a(z, y)$ which satisfy the hypotheses $H_{2}(a)$ :

(1) the map $a(z, y) \equiv a(y)=\|y\|^{p-2} y, 1<p<\infty$, corresponds to the $p$-Laplacian;

(2) $a(z, y)=\beta(z)\|y\|^{p-2} y$, with $1<p<\infty, \beta \in C^{1}(\bar{Z})$, and $\beta(z) \geq c_{10}>0$ for all $z \in \bar{Z}$;

(3) the map $a(z, y)=\beta(z)\left(1+\|y\|^{2}\right)^{\frac{p-2}{p}} y$, with $1<p<2, \beta \in C^{1}(\bar{Z})$, and $\beta(z) \geq c_{10}>0$ for all $z \in \bar{Z}$, corresponds to the weighted generalized mean curvature operator;

(4) $a(z, y)=\beta(z)\left(\|y\|^{p-2} y+\ln \left(1+\|y\|^{2}\right) y\right)$, with $1<p<\infty, \beta \in C^{1}(\bar{Z})$, and $\beta(z) \geq c_{10}>0$ for all $z \in \bar{Z}$;

(5) $a(z, y)=A(z) y$ with $A \in C^{1}\left(\bar{Z}, \mathbb{R}^{N \times N}\right), A(z)>0$ for all $z \in \bar{Z}$.

The hypotheses on the nonlinearity $f(z, x)$ are the following:

$H_{2}(f): f: Z \times \mathbb{R} \rightarrow \mathbb{R}$ is a function such that $f(z, 0)=0$ a.e. on $Z$, hypotheses $H_{2}(f)(i)-(v)$ are the same as the corresponding hypotheses $H_{1}(f)(\mathrm{i})-(\mathrm{v})$ and

(vi) (sign condition) for almost all $z \in Z$ and all $x \in \mathbb{R}, f(z, x) x \geq 0$.

Example 10 The following function $f(z, x) \equiv f(x)$ satisfies hypotheses $H_{2}(f)$ :

$$
f(x)= \begin{cases}|x|^{r-2} x & \text { if }|x| \leq 1, \\ p|x|^{p-2} x\left(\ln (|x|)+\frac{1}{p}\right) & \text { if }|x|>1,\end{cases}
$$

with $1<p<r<\infty$. 
As we already mentioned in the Introduction, our method of proof involves also truncation techniques. So, we introduce the following truncations.

Definition 10 Define the following truncations of the nonlinearity $f(z, \cdot)$ :

$$
f_{+}(z, x)=\left\{\begin{array}{ll}
0 & \text { if } x \leq 0 \\
f(z, x) & \text { if } x>0
\end{array} \quad \text { and } \quad f_{-}(z, x)=\left\{\begin{array}{ll}
f(z, x) & \text { if } x<0 \\
0 & \text { if } x \geq 0
\end{array} .\right.\right.
$$

Both functions $f_{+}$and $f_{-}$are Carathéodory functions (i.e., measurable in $z \in Z$ and continuous in $x \in \mathbb{R}$ ). We set

$$
F_{+}(z, x)=\int_{0}^{x} f_{+}(x, s) \mathrm{d} s \quad \text { and } \quad F_{-}(z, x)=\int_{0}^{x} f_{-}(x, s) \mathrm{d} s,
$$

and introduce the functionals $\varphi_{\lambda}^{ \pm}: W_{0}^{1, p}(Z) \rightarrow \mathbb{R}, \lambda>0$, defined by

$$
\varphi_{\lambda}^{ \pm}(x)=\int_{Z} G(z, D x) \mathrm{d} z-\lambda \int_{Z} F_{ \pm}(z, x) \mathrm{d} z \quad \text { for all } x \in W_{0}^{1, p}(Z) .
$$

Clearly, $\varphi_{\lambda}^{ \pm} \in C^{1}\left(W_{0}^{1, p}(Z)\right)$. $\varphi_{\lambda}^{ \pm}$

Arguing as in the proofs of Propositions 2 and 3, we obtain the corresponding results for the functionals

Proposition 5 If $\lambda>0$, hypotheses $H_{2}(a)$ and $H_{2}(f)$ hold, then the functionals $\varphi_{\lambda}^{ \pm}$satisfy the $C$ condition.

Proposition 6 If $\lambda>0$, hypotheses $H_{2}(a)$ and $H_{2}(f)$ hold, then $x=0$ is a local minimizer for the functionals $\varphi_{\lambda}^{ \pm}$.

Proposition 6 implies that we can find $\rho_{\lambda}>0$ small such that

$$
\varphi_{\lambda}^{ \pm}(0)=0<\inf \left\{\varphi_{\lambda}^{ \pm}(u):\|u\|=\rho_{\lambda}\right\}=c_{\lambda}^{ \pm}
$$

Moreover, using hypothesis $H_{2}(f)$ (iv) and arguing as in the proof of Proposition 4, we have the following result:

Proposition 7 If $\lambda>0$, hypotheses $H_{2}(a)$ and $H_{2}(f)$ hold, then

$$
\varphi_{\lambda}^{+}\left(t u_{1}\right) \rightarrow-\infty \quad \text { as } t \rightarrow+\infty
$$

and

$$
\varphi_{\lambda}^{-}\left(t u_{1}\right) \rightarrow-\infty \quad \text { as } \quad t \rightarrow-\infty .
$$

At this point, we are ready to produce the first two nontrivial solutions of problem $(P)_{\lambda}$ valid for all $\lambda>0$. These solutions have constant sign (one is positive and the other is negative).

Proposition 8 If hypotheses $H_{2}(a)$ and $H_{2}(f)$ hold, then, for every $\lambda>0$, problem $(P)_{\lambda}$ has two nontrivial solutions

$$
x_{0} \in \operatorname{int} C_{+} \quad \text { and } \quad v_{0} \in-\operatorname{int} C_{+} .
$$

ProOF. First we produce the positive solution.

Propositions 5, 7 and (39) permit the application of Theorem 1 (the mountain pass theorem). So, we can find $x_{0} \in W_{0}^{1, p}(Z), x_{0} \neq 0$ such that $\left(\varphi_{\lambda}^{+}\right)^{\prime}\left(x_{0}\right)=0$, thus

$$
S\left(x_{0}\right)=\lambda N_{+}\left(x_{0}\right)
$$


where $N_{+}(u)(\cdot)=f_{+}(\cdot, u(\cdot))$ for all $u \in W_{0}^{1, p}(Z)$. On (40), we act with $-x_{0}^{-} \in W_{0}^{1, p}(Z)$ and obtain

$$
\frac{c_{10}}{p-1}\left\|D x_{0}^{-}\right\|_{p}^{p} \leq 0, \quad \text { (see Lemma 1(c)) }
$$

which implies that $x_{0} \geq 0$ and $x_{0} \neq 0$.

Also, from (40), we have

$$
\left\{\begin{array}{l}
-\operatorname{div} a\left(z, D x_{0}(z)\right)=\lambda f_{+}\left(z, x_{0}(z)\right)=\lambda f\left(z, x_{0}(z)\right) \quad \text { a.e. on } Z, \\
\left.x_{0}\right|_{\partial Z}=0 .
\end{array}\right.
$$

As before, nonlinear regularity theory (namely, Theorem 7.1, p.286, of Ladyshenskaya-Uraltseva [16] and Theorem 1 of Lieberman [17]) implies that $x_{0} \in C_{+} \backslash\{0\}$. Moreover, hypotheses $H_{2}(f)$ (vi) (the sign condition) implies that

$$
\operatorname{div} a\left(z, D x_{0}(z)\right) \leq 0 \quad \text { a.e. on } Z \text {. }
$$

Invoking Theorem 3 of Montenegro [20] (see also Theorem 1.2 of Zhang [31]), we conclude that $x_{0} \in$ $\operatorname{int} C_{+}$.

Similarly, working with the functional $\varphi_{\lambda}^{-}$and using again the mountain pass theorem, we show that for every $\lambda>0$, problem $(P)_{\lambda}$ has another solution $v_{0} \in-\operatorname{int} C_{+}$.

Next, our effort will be to produce a third nontrivial solution, distinct from $x_{0}$ and $v_{0}$. We will do this using tools from Morse theory.

Proposition 9 If $\lambda>0$, hypotheses $H_{2}(a)$ and $H_{2}(f)$ hold, then

$$
C_{k}\left(\varphi_{\lambda}^{ \pm}, \infty\right)=0 \quad \text { for all } k \geq 0
$$

Proof. We do the proof for $\varphi_{\lambda}^{+}$, the proof for $\varphi_{\lambda}^{-}$being similar.

Let $\psi_{\lambda}^{+}=\left.\varphi_{\lambda}^{+}\right|_{C_{0}^{1}(\bar{Z})}$. Nonlinear regularity theory (see Ladyzhenskaya-Uraltseva [16, p. 286], and Lieberman [17, Theorem 1]), together with the nonlinear strong maximum principle (see Montenegro [20], Zhang [31], and Vazquez [30] for the particular case of the $p$-Laplacian), imply that the nontrivial critical points of $\varphi_{\lambda}^{+}$belong to int $C_{+}$and so the functionals $\psi_{\lambda}^{+}$and $\varphi_{\lambda}^{+}$have the same critical points. The space $C_{0}^{1}(\bar{Z})$ is dense in $W_{0}^{1, p}(Z)$. Hence, by virtue of Theorem 16 of Palais [23], we have

$$
H_{k}\left(W_{0}^{1, p}(Z),\left(\dot{\varphi}_{\lambda}^{+}\right)^{a}\right)=H_{k}\left(C_{0}^{1}(\bar{Z}),\left(\dot{\psi}_{\lambda}^{+}\right)^{a}\right) \quad \text { for all } a \in \mathbb{R} \text { and all } k \geq 0
$$

From Section 2, we know that for all $a<\inf \psi_{\lambda}^{+}(K)$, we have

$$
H_{k}\left(C_{0}^{1}(\bar{Z}),\left(\dot{\psi}_{\lambda}^{+}\right)^{a}\right)=C_{k}\left(\psi_{\lambda}^{+}, \infty\right)=H_{k}\left(C_{0}^{1}(\bar{Z}),\left(\psi_{\lambda}^{+}\right)^{a}\right) \quad \text { for all } k \geq 0,
$$

and

$$
H_{k}\left(W_{0}^{1, p}(Z),\left(\dot{\varphi}_{\lambda}^{+}\right)^{a}\right)=C_{k}\left(\varphi_{\lambda}^{+}, \infty\right)=H_{k}\left(W_{0}^{1, p}(Z),\left(\varphi_{\lambda}^{+}\right)^{a}\right) \quad \text { for all } k \geq 0 .
$$

From (41), (42) and (43), we see that it suffices to show that

$$
H_{k}\left(C_{0}^{1}(\bar{Z}),\left(\psi_{\lambda}^{+}\right)^{a}\right)=0 \quad \text { for all } k \geq 0
$$

To this end, we introduce the sets

$$
\begin{aligned}
\partial B_{1}^{c} & =\left\{x \in C_{0}^{1}(\bar{Z}):\|x\|_{C_{0}^{1}(\bar{Z})}=1\right\}, \\
\partial B_{1,+}^{c} & =\left\{x \in \partial B_{1}^{c}: x(z)>0 \quad \text { for some } z \in Z\right\} .
\end{aligned}
$$


Consider the map $h_{+}:[0,1] \times \partial B_{1,+}^{c} \rightarrow \partial B_{1,+}^{c}$ defined by

$$
h_{+}(t, x)=\frac{(1-t) x+t u_{1}}{\left\|(1-t) x+t u_{1}\right\|_{C_{0}^{1}(\bar{Z})}} \quad \text { for all }(t, x) \in[0,1] \times \partial B_{1,+}^{c} .
$$

Evidently, $h_{+}(t, x)$ is a continuous homotopy and $h(1, x)=\frac{u_{1}}{\left\|u_{1}\right\|_{C_{0}^{1}(\bar{Z})}} \in \partial B_{1,+}^{c}$. Therefore, the set $\partial B_{1,+}^{c}$ is contractible in itself.

Next, we will show that for $a<0$ with $|a|$ large, the set $\left(\dot{\psi}_{\lambda}^{+}\right)^{a}$ is of the same homotopy type of $\partial B_{1,+}^{c}$. Due to hypothesis $H_{2}(f)$ (iv), as in the proof of Proposition 4, for all $u \in \partial B_{1,+}^{c}$, we have

$$
\psi_{\lambda}^{+}(t u) \rightarrow-\infty \quad \text { as } t \rightarrow+\infty
$$

By virtue of hypothesis $H_{2}(f)$ (iv), we can find $\beta>0$ and $M_{7} \equiv M_{7}(\beta)>0$, such that

$$
f_{+}(z, x) x-p F_{+}(z, x) \geq \beta x^{\tau} \quad \text { for a.a. } z \in Z \text { and all } x \geq M_{7} .
$$

Hence, for all $x \in W_{0}^{1, p}(Z)$, we have

$$
\begin{aligned}
\int_{Z} p F_{+}(z, x) \mathrm{d} z & -\int_{Z} f_{+}(z, x) x \mathrm{~d} z \\
& =\int_{\left\{x \geq M_{7}\right\}} p F_{+}(z, x) \mathrm{d} z+\int_{\left\{x<M_{7}\right\}} p F_{+}(z, x) \mathrm{d} z \\
& -\int_{\left\{x \geq M_{7}\right\}} f_{+}(z, x) x \mathrm{~d} z-\int_{\left\{x<M_{7}\right\}} f_{+}(z, x) x \mathrm{~d} z \\
& \leq \int_{\left\{x \geq M_{7}\right\}} \beta x^{\tau} \mathrm{d} z+c_{11},
\end{aligned}
$$

for some $c_{11}>0$ (see (45) and recall that $f_{+}(z, x)=0=F_{+}(z, x)$ for a.a. $z \in Z$, all $x \leq 0$ ). Then, for $t>0$, we have

$$
\begin{aligned}
\frac{\mathrm{d}}{\mathrm{d} t} \psi_{\lambda}^{+}(t u) & =<\varphi_{\lambda}^{+}(t u), u> \\
& =t^{p-1}\|D u\|_{p}^{p}-\lambda \int_{Z} f_{+}(z, t u) \mathrm{d} z \\
& \leq \frac{1}{t}\left[t^{p}\|D u\|_{p}^{p}-\lambda \int_{Z} p F(z, t u) \mathrm{d} z+c_{11}\right] \\
& =\frac{1}{t}\left[p \psi_{\lambda}^{+}(t u)+c_{11}\right]
\end{aligned}
$$

which implies that

$$
\frac{\mathrm{d}}{\mathrm{d} t} \psi_{\lambda}^{+}(t u)<0
$$

for $t>0$ large such that $\psi_{\lambda}^{+}(t u)<-c_{11} p^{-1}$ (see (44)).

Let

$$
a<\min \left\{-\frac{c_{11}}{p}, \inf _{\bar{B}_{1}^{c}} \psi_{\lambda}^{+}\right\},
$$

recall $\bar{B}_{1}^{c}=\left\{x \in C_{0}^{1}(\bar{Z}):\|x\|_{C_{0}^{1}(\bar{Z})} \leq 1\right\}$. Then, we can find a unique $\vartheta(u) \geq 1$ such that

$$
\begin{cases}\psi_{\lambda}^{+}(t u)>a & \text { if } t \in[0, \vartheta(u)] \\ \psi_{\lambda}^{+}(t u)=a & \text { if } t=\vartheta(u) \\ \psi_{\lambda}^{+}(t u)<a & \text { if } t>\vartheta(u)\end{cases}
$$


Moreover, by virtue of the implicit function theorem, we have $\vartheta \in C\left(\partial B_{1,+}^{c},[1,+\infty)\right)$ and

$$
\left(\psi_{\lambda}^{+}\right)^{a}=\left\{t u: u \in \partial B_{1,+}^{c} \text { and } t \geq \vartheta(u)\right\}
$$

We set $E_{+}=\left\{t x: x \in \partial B_{1,+}^{c}\right.$ and $\left.t \geq 1\right\}$ and consider the map $\hat{h}_{+}:[0,1] \times E_{+} \rightarrow E_{+}$defined by

$$
\hat{h}_{+}(s, t u)= \begin{cases}(1-s) t u+s \vartheta(u) u & \text { if } 1 \leq t<\vartheta(u), \\ t u & \text { if } t \geq \vartheta(u)\end{cases}
$$

Evidently, $\hat{h}_{+}$is a continuous deformation. Moreover, we have

$$
\begin{gathered}
\qquad \hat{h}_{+}\left(1, E_{+}\right) \subseteq\left(\psi_{\lambda}^{+}\right)^{a} \\
\text { and }\left.\hat{h}_{+}(s, \cdot)\right|_{E_{+}}=\left.\mathrm{id}\right|_{E_{+}} \quad \text { for all } s \in[0,1] \quad \quad \text { (see (47)). }
\end{gathered}
$$

This shows that $\left(\psi_{\lambda}^{+}\right)^{a}$ is a strong deformation retract of $E_{+}$. Moreover, using the radial retraction, we see that the sets $E_{+}$and $\partial B_{1,+}^{c}$ are homotopic. Therefore, we have

$$
H_{k}\left(C_{0}^{1}(\bar{Z}),\left(\psi_{\lambda}^{+}\right)^{a}\right)=H_{k}\left(C_{0}^{1}(\bar{Z}), E_{+}\right)=H_{k}\left(C_{0}^{1}(\bar{Z}), \partial B_{1,+}^{c}\right) \quad \text { for all } k \geq 0 .
$$

Recall that $\partial B_{1,+}^{c}$ is contractible. Hence,

$$
\begin{array}{ccc}
H_{k}\left(C_{0}^{1}(\bar{Z}), \partial B_{1,+}^{c}\right)=0 & \text { for all } k \geq 0, \\
\Downarrow & \\
H_{k}\left(C_{0}^{1}(\bar{Z}),\left(\psi_{\lambda}^{+}\right)^{a}\right) & \text { for all } k \geq 0 \\
\Downarrow & \text { (see (48)), } \\
C_{k}\left(\varphi_{\lambda}^{+}, \infty\right)=0 \quad \text { for all } k \geq 0 . &
\end{array}
$$

Similarly, we may show that

$$
C_{k}\left(\varphi_{\lambda}^{-}, \infty\right)=0 \quad \text { for all } k \geq 0
$$

This proves the Proposition.

Proposition 10 If $\lambda>0$, hypotheses $H_{2}(a)$ and $H_{2}(f)$ hold, then

$$
C_{k}\left(\varphi_{\lambda}, \infty\right)=0 \quad \text { for all } k \geq 0
$$

ProOF. The proof of this result is analogous to the proof of Proposition 9.

So, let $u \in \partial B_{1}=\left\{u \in W_{0}^{1, p}(Z):\|u\|=1\right\}$. Then, as in the proof of Proposition 4, we have

$$
\varphi_{\lambda}(t u) \rightarrow-\infty \quad \text { as }|t| \rightarrow \infty .
$$

Hypothesis $H_{2}(f)\left(\right.$ iv) implies that we can find $\beta>0$ and $M_{8} \equiv M_{8}(\beta)>0$ such that

$$
f(z, x) x-p F(z, x) \geq \beta|x|^{\tau} \quad \text { for a.a. } z \in Z \text {, all }|x| \geq M_{8} .
$$

Thus, for all $x \in W_{0}^{1, p}(Z)$, we have

$$
\begin{aligned}
\int_{Z} p F(z, x) \mathrm{d} z-\int_{Z} f(z, x) \mathrm{d} z= & \int_{\left\{|x| \geq M_{8}\right\}} p F(z, x) \mathrm{d} z+\int_{\left\{|x|<M_{8}\right\}} p F(z, x) \mathrm{d} z \\
& -\int_{\left\{|x| \geq M_{8}\right\}} f(z, x) x \mathrm{~d} z-\int_{\left\{|x|<M_{8}\right\}} f(z, x) x \mathrm{~d} z \\
\leq & -\int_{\left\{|x| \geq M_{8}\right\}} \beta|x|^{\tau} \mathrm{d} z+c_{12} \quad \text { for some } c_{12}>0
\end{aligned}
$$


(see (50) and hypothesis $H_{2}(f)$ (iii)).

Let $\eta_{\lambda}=\lambda c_{12}+1$ and $\mu<-\frac{1}{p} \eta_{\lambda}<0$. By virtue of (49), for $t>0$ large, we have

$$
\varphi_{\lambda}(t u)=\frac{t^{p}}{p}-\lambda \int_{Z} F(z, t u) \mathrm{d} z \leq \mu \quad\left(\text { recall } u \in \partial B_{1}\right)
$$

We have

$$
\begin{aligned}
\frac{\mathrm{d}}{\mathrm{d} t} \varphi_{\lambda}(t u) & =<\varphi_{\lambda}^{\prime}(t u), u> \\
& =t^{p-1}-\lambda \int_{Z} f(z, t u) u \mathrm{~d} z \\
& =\frac{1}{t}\left[t^{p}-\lambda \int_{z} f(z, t u) t u \mathrm{~d} z\right] \\
& \leq \frac{1}{t}\left[p \mu+\lambda \int_{Z} p F(z, t u) \mathrm{d} z-\lambda \int_{Z} f(z, t u) t u \mathrm{~d} z\right] \\
& \leq \frac{1}{t}\left[p \mu+\lambda c_{12}\right] \\
& <0
\end{aligned}
$$

(recall the choice of $\mu<0$ ).

Therefore, as before (see the proof of Proposition 9), via the implicit function theorem, we can find a unique $\vartheta \in C\left(\partial B_{1}\right)$ such that

$$
\varphi_{\lambda}(\vartheta(u) u)=\mu \quad \text { for all } u \in \partial B_{1} .
$$

For $u \neq 0$, we set $\hat{\vartheta}(u)=\frac{1}{\|u\|} \vartheta\left(\frac{u}{\|u\|}\right)$. Evidently, $\hat{\vartheta} \in C\left(W_{0}^{1, p}(Z) \backslash\{0\}\right)$ and

$$
\varphi_{\lambda}(\hat{\vartheta}(u) u)=\mu \quad \text { for all } u \in W_{0}^{1, p}(Z), u \neq 0 .
$$

In addition, we have that $\varphi_{\lambda}(u)=\mu$ implies $\hat{\vartheta}(u)=1$. Hence, if we define

$$
\hat{\vartheta}_{0}(u)= \begin{cases}1 & \text { if } \varphi_{\lambda}(u)<\mu, \\ \hat{\vartheta}(u) & \text { if } \varphi_{\lambda}(u) \geq \mu,\end{cases}
$$

then, clearly, $\hat{\vartheta}_{0} \in C\left(W_{0}^{1, p}(Z) \backslash\{0\}\right)$.

Consider the map $h:[0,1] \times\left(W_{0}^{1, p}(Z) \backslash\{0\}\right) \rightarrow W_{0}^{1, p}(Z) \backslash\{0\}$, defined by

$$
h(t, u)=(1-t) u+t \hat{\vartheta}_{0}(u) u .
$$

Evidently, $h$ is a continuous homotopy. Moreover,

$$
h\left(1, W_{0}^{1, p}(Z) \backslash\{0\}\right) \subseteq \varphi_{\lambda}^{\mu} \quad \text { and }\left.\left.\quad h(t, \cdot)\right|_{\varphi_{\lambda}^{\mu}} \equiv \mathrm{id}\right|_{\varphi_{\lambda}^{\mu}} .
$$

This shows that $\varphi_{\lambda}^{\mu}$ is a strong deformation retract of $W_{0}^{1, p}(Z) \backslash\{0\}$. Also, using the radial retraction, we see that $\partial B_{1}$ is a retract of $W_{0}^{1, p}(Z) \backslash\{0\}$. Then, invoking Theorem 6.5, p. 325, of Dugundji [10], we have that

$\partial B_{1}$ is a deformation retract of $W_{0}^{1, p}(Z) \backslash\{0\}$,

$\Downarrow$

$\partial B_{1}$ is of the same homotopy type as $W_{0}^{1, p}(Z) \backslash\{0\}$. 
Also, we have that

$$
\varphi_{\lambda}^{\mu} \text { is of the same homotopy type as } W_{0}^{1, p}(Z) \backslash\{0\} .
$$

From (53) and (54), it follows that

$$
\begin{gathered}
\varphi_{\lambda}^{\mu} \text { is of the same homotopy type as } \partial B_{1}, \\
\Downarrow \\
H_{k}\left(W_{0}^{1, p}(Z), \varphi_{\lambda}^{\mu}\right)=H_{k}\left(W_{0}^{1, p}(Z), \partial B_{1}\right) \quad \text { for all } k \geq 0 .
\end{gathered}
$$

But, because $W_{0}^{1, p}(Z)$ is infinite dimensional, $\partial B_{1}$ is contractible in itself. Hence

$$
H_{k}\left(W_{0}^{1, p}(Z), \partial B_{1}\right)=0 \quad \text { for all } k \geq 0
$$

(see Granas-Dugundji [12, p. 389]), which implies that

$$
H_{k}\left(W_{0}^{1, p}(Z), \varphi_{\lambda}^{\mu}\right)=0 \quad \text { for all } k \geq 0 \quad \text { (see (55)). }
$$

So, if $\mu<\inf \varphi_{\lambda}(K)$, then from (56), we conclude that

$$
C_{k}\left(\varphi_{\lambda}, \infty\right)=0 \quad \text { for all } k \geq 0
$$

and this ends the proof.

Next, we calculate the critical groups of $\varphi_{\lambda}$ at the two nontrivial constant sign solutions $x_{0} \in \operatorname{int} C_{+}$ and $v_{0} \in-\operatorname{int} C_{+}$. We denote by $\delta_{i j}$ the Kronecker's delta function, i.e., $\delta_{i j}=1$ if $i=j$ and $\delta_{i j}=0$ if $i \neq j$.

Proposition 11 If $\lambda>0$, hypotheses $H_{2}(a)$ and $H_{2}(f)$ hold, then

$$
C_{k}\left(\varphi_{\lambda}, x_{0}\right)=C_{k}\left(\varphi_{\lambda}, v_{0}\right)=\delta_{k, 1} \mathbb{Z} \quad \text { for all } k \geq 0 .
$$

PROOF. We do the proof for $\left\{\varphi_{\lambda}, x_{0}\right\}$. The proof is similar for $\left\{\varphi_{\lambda}, v_{0}\right\}$.

Claim. $\quad C_{k}\left(\varphi_{\lambda}, x_{0}\right)=C_{k}\left(\varphi_{\lambda}^{+}, x_{0}\right)$ for all $k \geq 0$.

We consider the homotopy

$$
h^{\lambda}(t, x)=t \varphi_{\lambda}^{+}(x)+(1-t) \varphi_{\lambda}(x) \quad \text { for all } t \in[0,1] \text { and all } x \in W_{0}^{1, p}(Z)
$$

Arguing as in the proof of Proposition 2, we can easily check that for every $t \in[0,1], h_{t}^{\lambda}(\cdot)=h^{\lambda}(t, \cdot)$ satisfies the $C$-condition. Also, $x_{0} \in \operatorname{int} C_{+}$is a critical point of $h_{t}^{\lambda}$ for all $t \in[0,1]$.

We will show that, without any loss of generality, we may assume that the critical point $x_{0}$ of $h_{t}^{\lambda}$ is isolated uniformly in $t \in[0,1]$, i.e., we can find $r>0$ small such that $x_{0}$ is the only critical point of $\left\{h_{t}^{\lambda}(\cdot)\right\}_{t \in[0,1]}$ in

$$
\bar{B}_{r}\left(x_{0}\right)=\left\{x \in W_{0}^{1, p}(Z):\left\|x-x_{0}\right\| \leq r\right\} .
$$

We argue indirectly. So, suppose that this is not the case. We can find $\left\{t_{n}\right\}_{n \geq 1} \subset[0,1]$ and $\left\{x_{n}\right\}_{n \geq 1} \subset$ $W_{0}^{1, p}(Z)$ such that

$$
t_{n} \rightarrow t \text { in }[0,1], \quad x_{n} \rightarrow x_{0} \text { in } W_{0}^{1, p}(Z), \quad \text { and } \quad\left(h_{t_{n}}^{\lambda}\right)^{\prime}\left(x_{n}\right)=0 \text { for all } n \geq 1 .
$$

From (57), we have

$$
S\left(x_{n}\right)=\lambda t_{n} N_{+}\left(x_{n}\right)+\lambda\left(1-t_{n}\right) N\left(x_{n}\right) \quad \text { for all } n \geq 1
$$


which implies, for all $n \geq 1$,

$$
\left\{\begin{array}{l}
-\operatorname{div} a\left(z, D x_{n}(z)\right)=\lambda t_{n} f_{+}\left(z, x_{n}(z)\right)+\lambda\left(1-t_{n}\right) f\left(z, x_{n}(z)\right) \quad \text { a.e. on } Z, \\
\left.x_{n}\right|_{\partial Z}=0 .
\end{array}\right.
$$

Theorem 7.1, p. 286, of Ladyzhenskaya-Uraltseva [16], implies that we can find $M_{9}>0$ such that

$$
\left\|x_{n}\right\|_{\infty} \leq M_{9} \quad \text { for all } n \geq 1 .
$$

Because of (59), Theorem 1 of Lieberman [17] says that we can find $\eta \in(0,1)$ and $M_{10}>0$ such that

$$
x_{n} \in C_{0}^{1, \eta}(\bar{Z}) \quad \text { and } \quad\left\|x_{n}\right\|_{C_{0}^{1, \eta}(\bar{Z})} \leq M_{10} \quad \text { for all } n \geq 1 .
$$

We know that $C_{0}^{1, \eta}(\bar{Z})$ is embedded compactly in $C_{0}^{1}(\bar{Z})$. So, because of (60) and by passing to a subsequence, if necessary, we may assume that

$$
x_{n} \rightarrow x_{0} \quad \text { in } C_{0}^{1}(\bar{Z}) .
$$

Recall that $x_{0} \in \operatorname{int} C_{+}$(see Proposition 2). So, we can find $n_{0} \geq 1$ such that

$$
x_{n} \in C_{+} \quad \text { for all } n \geq n_{0} \quad \text { (see (61)). }
$$

Then from (58), we have, for all $n \geq n_{0}$,

$$
\left\{\begin{array}{l}
-\operatorname{div} a\left(z, D x_{n}(z)\right)=\lambda f\left(z, x_{n}(z)\right) \quad \text { a.e. on } Z, \\
\left.x_{n}\right|_{\partial Z}=0
\end{array}\right.
$$

which implies that $\left\{x_{n}\right\}_{n \geq n_{0}}$ is a whole sequence of distinct nontrivial solutions of $(P)_{\lambda}$ and so we are done.

Hence, we may assume that there is $r>0$ small such that $x_{0}$ is the only critical point of the family $\left\{h_{t}^{\lambda}\right\}_{t \in[0,1]}$ in $\bar{B}_{r}\left(x_{0}\right)$. Thus, invoking the homotopy invariance property of critical groups (see Chang [6, p. 4], and Mawhin-Willem [18, p. 169]), we have

$$
C_{k}\left(h_{0}^{\lambda}, x_{0}\right)=C_{k}\left(h_{1}^{\lambda}, x_{0}\right) \quad \text { for all } k \geq 0,
$$

and therefore

$$
C_{k}\left(\varphi_{\lambda}, x_{0}\right)=C_{k}\left(\varphi_{\lambda}^{+}, x_{0}\right) \quad \text { for all } k \geq 0 .
$$

This proves the Claim.

According to the Claim, to prove this Proposition, it suffices to compute $C_{k}\left(\varphi_{\lambda}^{+}, x_{0}\right)$ for all $k \geq 0$.

We assume that $\left\{v_{0}, 0, x_{0}\right\}$ are the only critical points $\varphi_{\lambda}$. Otherwise, we already have a third nontrivial solution of $(P)_{\lambda}$ and so we are done.

Let $\eta_{\lambda}^{+}=\varphi_{\lambda}^{+}\left(x_{0}\right)=\varphi_{\lambda}\left(x_{0}\right)$. We know that $0<c_{\lambda}^{+} \leq \eta_{\lambda}^{+}$(see (39)). Let $\xi<0$ and consider the following triple of sets

$$
\mathcal{V}_{\xi}=\left(\varphi_{\lambda}^{+}\right)^{\xi}, \quad \mathcal{V}_{\gamma}=\left(\varphi_{\lambda}^{+}\right)^{\gamma} \quad \text { and } \quad \mathcal{W}=W_{0}^{1, p}(Z)
$$

which satisfy

$$
\mathcal{V}_{\xi} \subseteq \mathcal{V}_{\gamma} \subseteq \mathcal{W} \quad \text { with } 0<\gamma<\eta_{\lambda}^{+}
$$

We consider the long exact sequence corresponding to this triple of sets. So, we have

$$
\cdots H_{k}\left(\mathcal{V}_{\gamma}, \mathcal{V}_{\xi}\right) \stackrel{i_{*}}{\longrightarrow} H_{k}\left(\mathcal{W}, \mathcal{V}_{\xi}\right) \stackrel{j_{*}}{\longrightarrow} H_{k}\left(\mathcal{W}, \mathcal{V}_{\gamma}\right) \stackrel{\partial_{*}}{\longrightarrow} H_{k-1}\left(\mathcal{V}_{\gamma}, \mathcal{V}_{\xi}\right) \cdots
$$


where $i_{*}, j_{*}$ are the induced homomorphisms from the corresponding inclusion maps and $\partial_{*}$ is the induced boundary homomorphism.

As in the proof of Proposition 8, we can check that all the nontrivial critical points of $\varphi_{\lambda}^{+}$belong to $\operatorname{int} C_{+}$. Since, we have assumed that $\left\{v_{0}, 0, x_{0}\right\}$ are the only critical points of $\varphi_{\lambda}$, it follows that $\left\{0, x_{0}\right\}$ are the only critical points of $\varphi_{\lambda}^{+}$. But 0 is the only critical value of $\varphi_{\lambda}^{+}$in $[\xi, \gamma]$ and $\varphi_{\lambda}^{+}$satisfies the $C$-condition, so

$$
H_{k}\left(\mathcal{V}_{\gamma}, \mathcal{V}_{\xi}\right)=C_{k}\left(\varphi_{\lambda}^{+}, 0\right) \quad \text { for all } k \geq 0
$$

(e.g., see Chang [6, p. 35]). Recall that the deformation theorem is still valid under the $C$-condition and so, the result of Chang holds with the $P S$-condition replaced by the $C$-condition. From Proposition 6, we know that $x=0$ is a local minimizer of $\varphi_{\lambda}^{+}$. Hence

$$
C_{k}\left(\varphi_{\lambda}^{+}, 0\right)=\delta_{k, 0} \mathbb{Z} \quad \text { for all } k \geq 0
$$

(see Chang [6, p. 33], and Mawhin-Willem [18, p. 175]). From (63) and (64), it follows that

$$
H_{k}\left(\mathcal{V}_{\gamma}, \mathcal{V}_{\xi}\right)=\delta_{k, 0} \mathbb{Z} \quad \text { for all } k \geq 0 .
$$

In a similar fashion, we show that

$$
H_{k-1}\left(\mathcal{V}_{\gamma}, \mathcal{V}_{\xi}\right)=\delta_{k-1,0} \mathbb{Z}=\delta_{k, 1} \mathbb{Z} \quad \text { for all } k \geq 0 .
$$

From the definition of critical groups at infinity (see Section 2.2), we have

$$
H_{k}\left(\mathcal{W}, \mathcal{V}_{\xi}\right)=C_{k}\left(\varphi_{\lambda}^{+}, \infty\right) \quad \text { for all } k \geq 0,
$$

which implies that

$$
H_{k}\left(\mathcal{W}, \mathcal{V}_{\xi}\right)=0 \quad \text { for all } k \geq 0 \quad \text { (see Proposition 9). }
$$

Recall that by hypothesis, $x_{0}$ is the only critical point of $\varphi_{\lambda}^{+}$with critical value in $[\gamma,+\infty)$. Hence

$$
H_{k}\left(\mathcal{W}, \mathcal{V}_{\gamma}\right)=C_{k}\left(\varphi_{\lambda}^{+}, x_{0}\right) \quad \text { for all } k \geq 0
$$

We focus on the end part of (62), i.e., $k=1$, since the rest is trivial. Then

$$
H_{1}\left(\mathcal{V}_{\gamma}, \mathcal{V}_{\xi}\right) \stackrel{i_{*}}{\longrightarrow} H_{1}\left(\mathcal{W}, \mathcal{V}_{\xi}\right) \stackrel{j_{*}}{\longrightarrow} H_{1}\left(\mathcal{W}, \mathcal{V}_{\gamma}\right) \stackrel{\partial_{*}}{\longrightarrow} H_{0}\left(\mathcal{V}_{\gamma}, \mathcal{V}_{\xi}\right)
$$

From the fundamental homomorphism theorem, we have

$$
\begin{aligned}
\operatorname{rank} C_{1}\left(\varphi_{\lambda}^{+}, x_{0}\right) & =\operatorname{rank}\left(\operatorname{ker} \partial_{*}\right)+\operatorname{rank}\left(\operatorname{Im} \partial_{*}\right) & & (\text { see }(68) \text { and }(69)) \\
& =\operatorname{rank}\left(\operatorname{Im} j_{*}\right)+\operatorname{rank}\left(\operatorname{Im} \partial_{*}\right) & & \text { (since (69) is exact) } \\
& \leq 0+1 & & \text { (see (67) and (66)). }
\end{aligned}
$$

But from the proof of Proposition 8, we know that $x_{0} \in \operatorname{int} C_{+}$is a critical point of $\varphi_{\lambda}^{+}$of mountain pass type. Hence,

$$
\begin{array}{rrr}
C_{1}\left(\varphi_{\lambda}^{+}, x_{0}\right) \neq 0 & & \text { (e.g., see Chang [6, p. 89]), } \\
& \Downarrow & \text { (see (70)), } \\
C_{k}\left(\varphi_{\lambda}^{+}, x_{0}\right)=\delta_{k, 1} \mathbb{Z} & \text { for all } k \geq 0 & \\
C_{k}\left(\varphi_{\lambda}, x_{0}\right)=\delta_{k, 1} \mathbb{Z}(P)_{\lambda} & \Downarrow \quad \text { for all } k \geq 0 & \text { (see the Claim). }
\end{array}
$$

In a similar fashion, we also show that

$$
C_{k}\left(\varphi_{\lambda}, v_{0}\right)=\delta_{k, 1} \mathbb{Z} \quad \text { for all } k \geq 0 .
$$

This ends the proof of the Proposition.

We are now ready for the multiplicity theorem for problem $(P)_{\lambda}$ valid for all $\lambda>0$. 
Theorem 4 If hypotheses $H_{2}(a)$ and $H_{2}(f)$ hold, then, for every $\lambda>0$, problem $(P)_{\lambda}$ hast at least three nontrivial smooth solutions

$$
x_{0} \in \operatorname{int} C_{+}, \quad v_{0} \in-\operatorname{int} C_{+}, \quad \text { and } \quad y_{0} \in C_{0}^{1}(\bar{Z}) .
$$

ProOF. From Proposition 8, we already have two constant sign solutions

$$
x_{0} \in \operatorname{int} C_{+} \quad \text { and } \quad v_{0} \in-\operatorname{int} C_{+} .
$$

Suppose that $\left\{v_{0}, 0, x_{0}\right\}$ are the only critical points of $\varphi_{\lambda}$. From Proposition 11, we have

$$
C_{k}\left(\varphi_{\lambda}, x_{0}\right)=C_{k}\left(\varphi_{\lambda}, v_{0}\right)=\delta_{k, 1} \mathbb{Z} \quad \text { for all } k \geq 0 .
$$

Also, from Proposition 3, we know that $x=0$ is a local minimizer of $\varphi_{\lambda}$. Therefore,

$$
C_{k}\left(\varphi_{\lambda}, 0\right)=\delta_{k, 0} \mathbb{Z} \quad \text { for all } k \geq 0 .
$$

Finally, from Proposition 10, we have

$$
C_{k}\left(\varphi_{\lambda}, \infty\right)=0 \quad \text { for all } k \geq 0 .
$$

Note that (71)-(73) imply that $P\left(t, v_{0}\right)=P\left(t, x_{0}\right)=t, P(t, 0)=1$ and $P(t, \infty)=0$. Then, from the Morse relation (5) and assuming $Q(t)=\sum_{i=0}^{\infty} q_{i} t^{i}$ with $q_{i} \geq 0$, we have

$$
2 t+1=(1+t) Q(t) \Longleftrightarrow 0=\left(q_{0}-1\right)+\left(q_{1}+q_{0}-2\right) t+\left(q_{2}+q_{1}\right) t^{2}+\sum_{i=3}^{\infty}\left(q_{i}+q_{i-1}\right) t^{i},
$$

so $q_{0}=q_{1}=1$ and $q_{2}=-1<0$, which is a contradiction. This shows that $\varphi_{\lambda}$ has a third nontrivial critical point $y_{0}$ distinct from $\left\{v_{0}, x_{0}\right\}$. Hence, $y_{0}$ is a nontrivial solution of the problem $(P)_{\lambda}$ and nonlinear regularity theory implies $y_{0} \in C_{0}^{1}(\bar{Z})$.

Acknowledgement. The second author acknowledges the partial financial support from the Portuguese Foundation for Science and Technology (FCT), under the fellowship SFRH/BPD/38436/2007 and the research unit Mathematics and Applications.

\section{References}

[1] Ambrosetti, A., Brezis, H. And Cerami, G., (1994). Combined effects of concave and convex nonlinearities in some elliptic problems, J. Funct. Anal., 122, 519-543.

[2] Ambrosetti, A. And Rabinowitz, P., (1973). Dual variational methods in the critical point theory and applications, J. Funct. Anal., 14, 349-381.

[3] Bartolo, P., Berti, V. And Fortunato, D., (1983). Abstract critical point theorems and applications to some nonlinear problems with strong resonance at infinity, Nonlin. Anal., 7, 981-1012.

[4] BARTSCH, T. AND LI, S., (1997). Critical point theory for asymptotically quadratic functionals and applications to problems with resonance, Nonlin. Anal., 28, 419-441.

[5] Browder, F., (1970). Nonlinear elliptic boundary value problems and the generalized topological degree, Bull. Amer. Math. Soc, 76, 999-1005.

[6] Chang, K. C., (1993). Infinite Dimensional Morse Theory and Multiple Solution Problems, Birkhäuser, Boston.

[7] Corvellec, J.-N., (2001). On the second deformation lemma, Topol. Meth. Nonlin. Anal. 17, 55-66.

[8] Costa, D. and Magalhaes, C., (1995). Existence results for perturbations of the p-Laplacian, Nonlin. Anal., 24, 409-418. 
[9] De Napoli, P. And Mariani, M. C., (2003). Mountain pass solutions to equations of $p$-Laplacian, Nonlin. Anal., 54, 1205-1219.

[10] Dugundu, J., (1966). Topology, Allyn and Bacon Inc., Boston.

[11] Gasinski, L. and Papageorgiou, N. S., (2006). Nonlinear Analysis, Chapman \& Hall/CRC, Boca Raton.

[12] Granas, A. And Dugunsui, J., (2003). Fixed Point Theory, Springer-Verlag, New York.

[13] Guo, Z., (1996). On the number of positive solutions for quasilinear elliptic problems, Nonlin. Anal., 27, 229247.

[14] Guo, Z. And WebB, J. R. L., (2002). Large and small solutions of a class of quasilinear elliptic eigenvalue problems, J. Differential Equations, 180, 1-50.

[15] HAI, D. D., (2003). On a class of sublinear quasilinear elliptic problems, Proceedings AMS, 131, 2409-2414.

[16] Ladyzhenskaya, O. And Uraltseva, N., (1968). Linear and Quasilinear Elliptic Equations, Academic Press, New York.

[17] Lieberman, G., (1988). Boundary regularity for solutions of degenerate elliptic equations, Nonlin. Anal., 12, $1203-1219$.

[18] Mawhin, J. And Willem, M., (1989). Critical Point Theory and Hamiltonian Systems, Springer-Verlag, New York.

[19] MiYagaki, O. And Souto, M. A. S., Superlinear problems without Ambrosetti and Rabinowitz growth condition, J. Differential Equations, - in press.

[20] Montenegro, M., (1999). Strong maximum principles for supersolutions of quasilinear elliptic equations, Nonlin. Anal., 37, 431-448.

[21] Motreanu, D., Motreanu, V. and Papageorgiou, N. S., (2007). Multiple nontrivial solutions for nonlinear eigenvalue problems, Proceedings AMS, 135, 3649-3658.

[22] Motreanu, D., Motreanu, V. and Papageorgiou, N. S., (2007). A degree theoretic approach for multiple solutions of constant sign for nonlinear elliptic equations, Manuscripta Math., 124, 507-531.

[23] Palais, R., (1966). Homotopy theory of finite dimensional manifolds, Topology, 5, 1-16.

[24] Papageorgiou, N. S., Rocha, E. M. And Staicu, V., (2008). A multiplicity theorem for hemivariational inequalities with a $p$-Laplacian-like differential operator, Nonlin. Anal., 69, 1150-1163.

[25] Papageorgiou, N. S., Rocha, E. M. and Staicu, V., (2008). Multiplicity theorems for superlinear elliptic problems, Calc. Variations PDEs, 33, 199-230.

[26] Perera, K., (1997). Critical groups of pairs of critical points produced by linking subsets, J. Differential Equations, 140:1, 142-160.

[27] Perera, K., (2003). Multiple positive solutions for a class of quasilinear elliptic boundary value problems, Electronic J. Differential Equations, 7, 1-5.

[28] Schechter, M. And Zou, W., (2004). Superlinear problems, Pacific J. Math., 214, 145-160.

[29] Ubilla, P., (1999). Existence of nonnegative solutions for a quasilinear Dirichlet problem, Comm. Appl. Nonlinear Anal., 6:2, 89-99.

[30] VAzQuez, J., (1984). A strong maximum principle for some quasilinear elliptic equations, Appl. Math. Optim., 12, 191-202.

[31] ZHANG, Q., (2005). A strong maximum principle for differential equations with nonstandard $p(x)$-growth conditions, J. Math. Anal. Appl., 312, 24-32.

[32] ZHOU, H. S., (2002). An application of a mountain pass theorem, Acta Math. Sinica, 18, 27-36.

Nikolaos S. Papageorgiou

Department of Mathematics,

National Technical University,

Zografou Campus,

Athens 15780, Greece

npapg@math.ntua.gr
Eugénio M. Rocha

Department of Mathematics, University of Aveiro, 3810 - 194 Aveiro, Portugal eugenio@ua.pt 